\title{
Theoretical Expressions for the Ascent Rate of Moist Deep Convective Thermals
}

\author{
HUGH MORRISON \\ National Center for Atmospheric Research, Boulder, Colorado \\ JOHN M. PETERS \\ Department of Meteorology, U.S. Naval Postgraduate School, Monterey, California
}

(Manuscript received 4 October 2017, in final form 16 February 2018)

\begin{abstract}
An approximate analytic expression is derived for the ratio $\lambda$ of the ascent rate of moist deep convective thermals and the maximum vertical velocity within them; $\lambda$ is characterized as a function of two nondimensional buoyancy-dependent parameters $y$ and $h$ and is used to express the thermal ascent rate as a function of the buoyancy field. The parameter $y$ characterizes the vertical distribution of buoyancy within the thermal, and $h$ is the ratio of the vertically integrated buoyancy from the surface to the thermal top and the vertical integral of buoyancy within the thermal. Theoretical $\lambda$ values are calculated using values of $y$ and $h$ obtained from idealized numerical simulations of ascending moist updrafts and compared to $\lambda$ computed directly from the simulations. The theoretical values of $\lambda \approx 0.4-0.8$ are in reasonable agreement with the simulated $\lambda$ (correlation coefficient of 0.86 ). These values are notably larger than the $\lambda=0.4$ from Hill's (nonbuoyant) analytic spherical vortex, which has been used previously as a framework for understanding the dynamics of moist convective thermals. The relatively large values of $\lambda$ are a result of net positive buoyancy within the upper part of thermals that opposes the downward-directed dynamic pressure gradient force below the thermal top. These results suggest that nonzero buoyancy within moist convective thermals, relative to their environment, fundamentally alters the relationship between the maximum vertical velocity and the thermal-top ascent rate compared to nonbuoyant vortices. Implications for convection parameterizations and interpretation of the forces contributing to thermal drag are discussed.
\end{abstract}

\section{Introduction}

Since the 1950s, two primary conceptual models have served as a basis for understanding the structure and behavior of atmospheric moist convection [see the reviews of de Rooy et al. (2013) and Yano (2014)]. The first of these is the steady-state entraining plume model, which has a continuous buoyancy source and entrains steadily as buoyant air rises within the plume (e.g., Morton et al. 1956; Morton 1957). The second is the thermal model, which consists of a nonsteady, entraining buoyant bubble rising through a fluid (e.g., Scorer and Ludlam 1953; Levine 1959; Turner 1963). The plume and thermal models predict markedly different structure and flow characteristics. While there was some evidence at the time for the nonsteady thermal-like behavior of real moist convective updrafts, the earliest convection schemes were based on

Corresponding author: H. Morrison, morrison@ucar.edu the steady-state plume model (e.g., Simpson and Wiggert 1969), presumably because it was straightforward to adapt and implement. Most current schemes still utilize this framework (de Rooy et al. 2013; Yano 2014).

An early conceptual model of atmospheric moist convection occurring as thermals or "bubbles" of rising air was introduced by Scorer and Ludlam (1953) and Ludlam and Scorer (1953). This concept was extended by Levine (1959), who applied the mathematical framework of Hill's analytic spherical vortex (Hill 1894; Lamb 1932) to quantify moist thermal behavior. This model was expanded by Turner (1964) to account for increases in the size of entraining thermals as they rise. Several observational studies later reinforced the view of moist convection as consisting of a series of rising bubbles or a rising bubble with a trailing turbulent wake (e.g., Austin et al. 1985; Blyth et al. 1988; Blyth and Latham 1993; Damiani et al. 2006).

More recently, large-eddy simulation (LES) models have been used to investigate detailed aspects of moist 
convective behavior and structure (e.g., Siebesma and Cuijpers 1995; Siebesma and Jonker 2000; Stevens et al. 2001; Neggers et al. 2002; Bryan et al. 2003; Zhao and Austin 2005; Jonker et al. 2008; Heus et al. 2009; Sherwood et al. 2013; Romps and Charn 2015; Hernandez-Deckers and Sherwood 2016; Moser and Lasher-Trapp 2017). These LES studies have generally supported the view of moist convective updrafts being composed of a series of quasi-spherical bubbles of rising air (e.g., Sherwood et al. 2013; Romps and Charn 2015; Hernandez-Deckers and Sherwood 2016; Moser and Lasher-Trapp 2017). This nonsteady rising thermal view of convective structure challenges the steady-state plume framework assumed by most convection schemes. Recent papers have discussed contrasts between these conceptual models and implications for convection schemes (see Sherwood et al. 2013; de Rooy et al. 2013; Yano 2014). Thus, a better understanding of thermal dynamics is of practical importance for improving cumulus parameterizations.

Quantifying the ascent rate of moist convective thermals ${ }^{1}$ is important since it directly affects their ability to vertically transport mass, momentum, thermal energy, and water. From dimensional analysis, studies have related the thermal ascent (or descent) rate $w_{t}^{*}$ to the buoyant forcing via an empirical, nondimensional Froude number $F_{r}$ equal to the ratio of inertia and buoyancy forcing. Malkus and Scorer (1955) and Scorer (1957) defined $F_{r}$ using the relationship $w_{t}^{* 2}=F_{r}^{2} \bar{B} R$, where $\bar{B}$ is the thermalaveraged buoyancy and $R$ is the thermal radius; $B \equiv-g\left[\left(\rho-\rho_{0}\right) / \rho_{0}\right]$, where $\rho$ is the fluid density, $\rho_{0}$ is a reference state density taken to be the density of the fluid surrounding the thermal, and $g$ is the acceleration of gravity. These studies obtained $F_{r}$ of $O(1)$ from cloud and laboratory thermal observations. Thermal velocity was further explored in the laboratory study of Sanchez et al. (1989). They examined the ratio of $w_{t}^{*}$ and a scaling velocity $v_{s} \equiv\left(\bar{B} D_{0}\right)^{1 / 2}$, where $D_{0}$ is the initial thermal diameter. This ratio is analogous to $F_{r}$ as defined above except $R$ is replaced by $D_{0}$, and thus, it differs from $F_{r}$ by a factor of $2^{-1 / 2}$. They found $w_{t}^{*} / v_{s} \sim 0.5-0.8$ just after release of the thermals, consistent with $F_{r} \sim 1$ from Malkus and Scorer (1955) and Scorer (1957), with a decrease to $\sim 0.2-0.4$ as the thermals traveled distances greater than $5 D_{0}$ [see Fig. 8 in Sanchez et al. (1989)].

Studies have also characterized the ascent rate by the ratio $\lambda$ of $w_{t}^{*}$ and the maximum $w$ within the thermal

\footnotetext{
${ }^{1}$ Throughout the paper, "ascent rate" of thermals refers to the rate of ascent of the thermal top unless stated otherwise.
}

(e.g., Woodward 1959; Levine 1959; Romps and Charn 2015). In contrast to $w_{t}^{*}$, the maximum $w$ is associated with a thermal's internal toroidal circulation that does not directly contribute to net vertical transport of updraft properties. Nonetheless, recent studies have focused on the maximum $w$ within updrafts since it is well characterized by the forcing from the effective buoyancy (Peters 2016; Jeevanjee 2017), which is the sum of the buoyancy and the vertical buoyant perturbation pressure gradient forces (e.g., Davies-Jones 2003; Jeevanjee and Romps 2016). Thus, the thermal ascent rate can be obtained from $\lambda$ and the effective buoyancy if they are known. The latter can be directly calculated from the buoyancy field or derived from the vertical profile of the buoyancy and updraft radius following the approximations in Morrison (2016a), Peters (2016), Jeevanjee and Romps (2016), or Jeevanjee (2017).

Earlier laboratory tank experiments of thermals in a neutrally stratified fluid showed that $1 / \lambda=2.2(\lambda \approx 0.45)$ (Woodward 1959). Turner (1962) performed tank experiments on buoyant starting plumes ${ }^{2}$ and found that the mean ratio of the velocity of the thermal-like cap front at the plume head and the maximum velocity within the plume, analogous to $\lambda$, was 0.61 . More recent LES studies have reinforced these findings (Zhao and Austin 2005; Romps and Charn 2015). Zhao and Austin (2005) found that the mean thermal velocity was about half the local maximum vertical velocity within shallow convective thermals. A more detailed analysis of $\lambda$ from an LES of a field of scattered deep convection by Romps and Charn (2015) showed an average value of 0.56.

A key factor controlling $\lambda$ is the dynamic perturbation pressure, which is closely related to the vorticity field. A theoretical model for this pressure and flow field is provided by Hill's analytic spherical vortex (Hill 1894; Lamb 1932), first applied by Levine (1959) and more recently by Sherwood et al. (2013) and Romps and Charn (2015) as a framework for understanding moist convective dynamics. Hill's vortex has a maximum $w$ located in the vortex center, a downward-directed pressure gradient force between the center and top, and vertical symmetry in the thermal-relative flow about a horizontal axis through the vortex center. These features are broadly in line with observations of laboratory thermals (Scorer 1957; Woodward 1959) and

\footnotetext{
${ }^{2}$ Turner (1962) formulated the "starting plume" model by combining the thermal and plume models. This model consists of a rising buoyant plume, with the structure at the plume head consistent with a rising thermal and the region below described by the steady-state plume model.
} 
LESs of moist deep convective updraft structures (Sherwood et al. 2013; Romps and Charn 2015; Hernandez-Deckers and Sherwood 2016; Moser and Lasher-Trapp 2017). Hill's vortex gives an analytic value of $\lambda=0.4$ (see section 2a). This is notably smaller than the experimental and numerically simulated $\lambda \approx$ 0.45-0.61 for buoyant thermals (Woodward 1959; Turner 1962; Zhao and Austin 2005; Romps and Charn 2015), with some important consequences. For example, the value of $\lambda=0.56$ from Romps and Charn (2015) implies a mean ascent rate of moist thermals $40 \%$ greater than that for Hill's vortex for a given maximum $w$. This has implications for the effectiveness of moist thermals in driving the vertical mixing of updraft properties. Thus, while Hill's vortex is consistent with many features of moist thermal behavior, it does not provide very good accuracy in predicting $\lambda$.

Levine (1959) suggested that departures of $\lambda$ from Hill's value of 0.4 for moist thermals in the real atmosphere could arise from viscous effects, nonspherical thermal shape, or "small irregularities." The effects of stable stratification in the surrounding fluid are also neglected, which could impact $\lambda$, although Warren (1960) showed that wave drag is negligible for thermals except when their buoyancy changes sign. This result was supported by the LES study of Hernandez-Deckers and Sherwood (2016), while Romps and Oktem (2015) showed that wave drag may be important for large thermals (radius of $1 \mathrm{~km}$ ), especially those with a moderate ascent rate $\left(\sim 4 \mathrm{~m} \mathrm{~s}^{-1}\right)$. While these factors may play a role, we argue that a more fundamental inconsistency arises when applying Hill's vortex to moist thermal dynamics because Hill's vortex is nonbuoyant. One straightforward effect of buoyancy is an apparent "drag" on thermals induced by the buoyant perturbation pressure gradient force (Romps and Charn 2015; HernandezDeckers and Sherwood 2016). Herein, we show that another important effect of buoyancy is on $\lambda$ and the thermal-top ascent rate. It is proposed that the underestimation of $\lambda$ for moist thermals by Hill's vortex model compared to experimental and simulated values is a direct consequence of the positive buoyancy within thermals. This effect is quantified by deriving simple theoretical expressions for $\lambda$ and the thermal ascent rate as functions of the updraft buoyancy structure, extending Hill's analytic vortex solution. The primary goal is to improve the quantitative understanding of the factors controlling $\lambda$ and the ascent rate of convective thermals in a simplified framework, with a focus on moist deep convection. These expressions are compared to idealized three-dimensional (3D) numerical updraft simulations. Finally, we discuss implications for the drag force experienced by thermals and convection parameterizations.

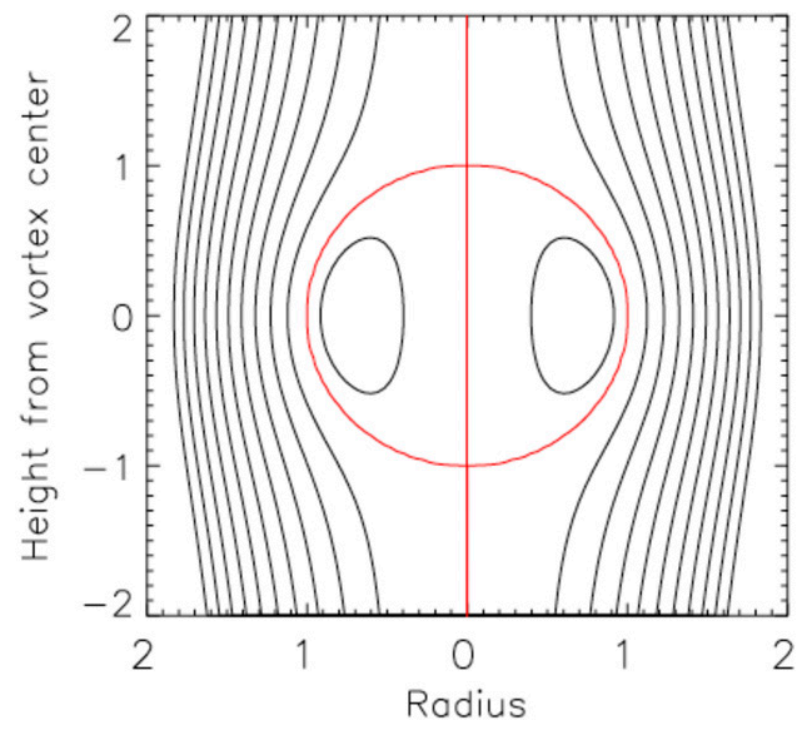

FIG. 1. Streamfunction isolines for Hill's analytic spherical vortex. The radius, height, and flow velocity are normalized such that the vortex radius is 1 and the flow velocity at the vortex top $w_{t}^{*}$ is 1 . Normalized isolines are shown with an interval of 0.1 between -1 and 1 , with the $\psi=0$ isoline shown in red.

The rest of the paper is organized as follows: Section 2 presents an overview of Hill's analytic vortex and derivations of the theoretical expressions for $\lambda$ and moist convective thermal-top ascent rate. Section 3 describes the configuration of the numerical simulations. The theoretical expressions for $\lambda$ and ascent rate are compared with values from the numerical simulations in section 4. The relationship to previous studies on thermal drag is discussed in section 5. Implications for convection parameterization are discussed in section 6 . Finally, section 7 gives a summary and conclusions.

\section{Theory}

\section{a. Flow and perturbation pressure in Hill's vortex}

Hill's analytic spherical vortex (Hill 1894) is steady state and translates with velocity $U=w_{t}^{*}$ through an inviscid fluid that is at rest infinitely far from the vortex ( $w_{t}^{*}$ is the velocity at the vortex top in this frame of reference). This flow structure is shown in Fig. 1. The motion of the entire field is axisymmetric around the line of forward motion of the vortex. Vorticity within the sphere is proportional to the distance from the axis of symmetry, while the flow outside the sphere is the same as the irrotational motion around a moving solid sphere. Matching solutions for the interior and exterior flow, the streamfunction in cylindrical axisymmetric coordinates $(r, z)$ is given by (Hill 1894; Lamb 1932) 
$\psi=\frac{3}{4} w_{t}^{*} r^{2}-\frac{3}{4} w_{t}^{*} \frac{r^{2}}{a^{2}}\left(z^{2}+r^{2}\right), \quad$ if $\quad z^{2}+r^{2} \leq a^{2}$

$\psi=\frac{1}{2} w_{t}^{*} r^{2} a^{3}\left(z^{2}+r^{2}\right)^{-3 / 4}-\frac{1}{2} w_{t}^{*} r^{2}, \quad$ otherwise,

where $a$ is the radius of the vortex (Fig. 1).

The velocity along $r=0$ within the vortex in the frame of reference following the vortex (such that the velocity at the vortex top is zero) is

$$
w_{r=0}=\frac{1}{r} \frac{\partial \psi}{\partial r}=\frac{3}{2} w_{t}^{*}\left(1-\frac{z^{2}}{a^{2}}\right),
$$

for $z^{2}+r^{2} \leq a^{2}$. Hereafter, $w^{*}$ indicates vertical velocity in the frame of reference with zero velocity infinitely far from the vortex, while $w$ indicates vertical velocity in the frame of reference with zero velocity at the vortex top (i.e., following the vortex).

Defining $\lambda \equiv w_{t}^{*} / w_{m}^{*}$, where $w_{m}^{*}$ is the maximum vertical velocity in the vortex, $\lambda=0.4$ can be obtained from (2) using the relation $w_{m}=w_{m}^{*}-w_{t}^{*}$, where $w_{m}$ is the maximum vertical velocity at the vortex center $(r=0$, $z=0)$ in the frame of reference following the vortex. The pressure difference $\Delta p$ between the vortex top $(z=a)$ and vortex center $(z=0)$ along $r=0$ as a function of $w_{m}^{*}$ and $\lambda$ can be derived as follows. First, an expression for $w_{m}$ is found by evaluating (2) at $z=0$; squaring this expression gives

$$
w_{m}^{2}=\frac{9}{4} w_{t}^{* 2} .
$$

Next, we integrate the Boussinesq vertical momentum equation in cylindrical axisymmetric coordinates, given by (6) below, over $d z$ from $z=0$ to $z=a$, assuming steady state (i.e., $\partial / \partial t=0$ ), noting that $u=0$ by symmetry and buoyancy $B=0$ since the vortex is nonbuoyant. This gives an expression relating $\Delta p$ and $w_{m}^{2}$, which is combined with (3) to yield an expression for $\Delta p$ as a function of $w_{t}^{* 2}$. This is combined with $w_{t}^{* 2}=\lambda^{2} w_{m}^{* 2}$, which is derived from the definition of $\lambda$, yielding after rearranging terms,

$$
\Delta p=\frac{9}{8} \lambda^{2} \rho_{0} w_{m}^{* 2} .
$$

In general, the perturbation pressure field in a fluid can be expressed as $p=p_{\text {dynam }}+p_{\text {buoy }}$, where $p_{\text {dynam }}$ is the dynamic perturbation pressure and $p_{\text {buoy }}$ is the buoyant perturbation pressure relative to a motionless, hydrostatically balanced reference state. Here, we consider dynamic and buoyant perturbation pressures in the usual way, defined for Boussinesq flow as

$$
\begin{aligned}
\nabla^{2} p_{\text {dynam }} & =-\rho_{0} \nabla \cdot(\mathbf{u} \cdot \nabla \mathbf{u}) \text { and } \\
\nabla^{2} p_{\text {buoy }} & =\rho_{0} \frac{\partial B}{\partial z},
\end{aligned}
$$

where $\mathbf{u}$ is the wind vector. There is no buoyancy in Hill's vortex, and hence, $p_{\text {buoy }}=0$, and (4) gives the dynamic pressure difference between the vortex top and center.

\section{b. Ascent rate of a buoyant thermal}

Approximate analytic expressions for $\lambda$ and the ascent rate of a moist thermal that is buoyant with respect to its surrounding environment are derived next. Figure 2 shows the conceptual model that serves as the basis for this derivation, where the structure of the dynamic and buoyant perturbation pressure forcings are based on Peters (2016) and Morrison (2016a), respectively, as well as the numerical simulations presented in section 4 . We start with the inviscid Boussinesq vertical momentum equation in axisymmetric cylindrical coordinates:

$$
\frac{\partial w}{\partial t}+u \frac{\partial w}{\partial r}+w \frac{\partial w}{\partial z}=-\frac{1}{\rho_{0}} \frac{\partial p}{\partial z}+B
$$

where $u$ is the radial velocity, $z$ is height, and $r$ is the horizontal distance from the updraft center. The quantities $p$ and $B$ are perturbation pressure and buoyancy relative to a motionless, hydrostatically balanced horizontally and temporally invariant reference state.

We focus on the thermal center at $r=0$, where $u=0$ by symmetry, and assume steady-state flow within the thermal relative to its ascent. Considering (6) in the frame of reference relative to the thermal ascent rate such that $w$ at the thermal top is zero $\left(w_{t}=0\right)$, vertical integration from the height of the thermal-relative maximum $w\left(w_{m}\right)$ to the thermal top gives

$$
w_{t}^{2}-w_{m}^{2}=-w_{m}^{2}=-\frac{2 \Delta p}{\rho_{0}}+2 \int_{H_{m}}^{H_{t}} B d z,
$$

where $H_{m}$ is the height of maximum vertical velocity, and $H_{t}$ is the height of the thermal top. Next, we introduce a nondimensional flow-dependent parameter $b$ defined by the ratio

$$
b \equiv \frac{w_{m}^{2}}{2 \bar{B} \Delta H},
$$

where $\Delta H=H_{t}-H_{b}$ and $\bar{B} \equiv(1 / \Delta H) \int_{H_{b}}^{H_{t}} B d z ; H_{b}$ is the height of the thermal bottom, and $b$ will be discussed in more detail later. We introduce another parameter $y$ defined by 


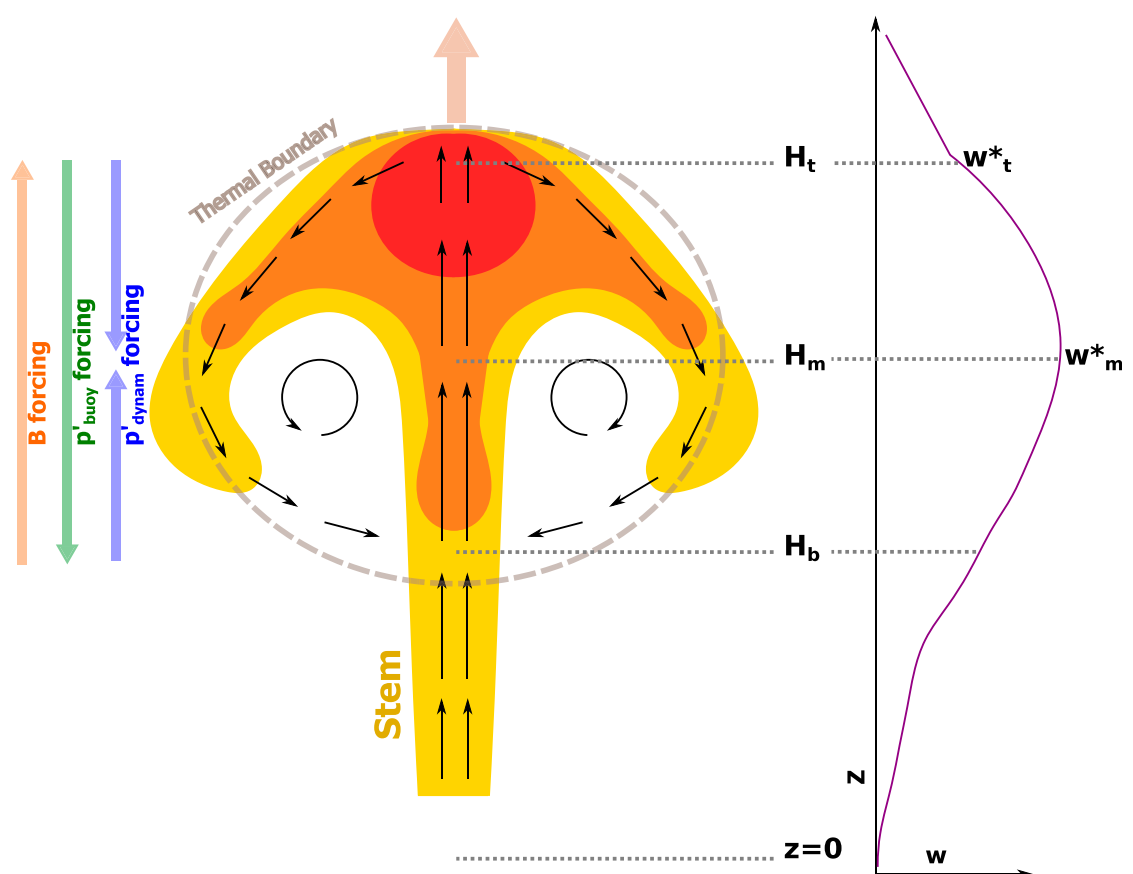

FIG. 2. Schematic of the buoyancy (yellow-orange shading) and flow (black arrows) in a rising moist thermal. (left) The general orientation of vertical accelerations in the thermal and (right) a hypothetical vertical velocity profile through the thermal center. Here, $w_{b}^{*}, w_{m}^{*}$, and $w_{t}^{*}$ are the vertical velocities at the thermal bottom, the maximum within the thermal, and the thermal top (at heights $H_{b}, H_{m}$, and $H_{t}$, respectively) in the frame of reference with $w^{*}=0$ at the surface.

$$
y \equiv 1-\frac{1}{\bar{B} \Delta H} \int_{H_{m}}^{H_{t}} B d z
$$

Physically, $y$ is the fraction of the thermal's total vertically integrated buoyancy contained between $H_{b}$ and $H_{m}$, and hence, $(1-y)$ is the fraction of the vertically integrated buoyancy between $H_{m}$ and $H_{t}$. Combining (7)-(9) gives

$$
-2 b \bar{B} \Delta H=-\frac{2 \Delta p}{\rho_{0}}+2(1-y) \bar{B} \Delta H .
$$

In contrast with (4) from Hill's vortex, $\Delta p$ in (10) for moist convective thermals includes both $p_{\text {dynam }}$ and $p_{\text {buoy }}$ (Fig. 2). Analytic approximations for $p_{\text {buoy }}$ were previously derived by Morrison (2016a). For 3D flow, the expression from Morrison [2016a, their (28)] gives an estimate for the difference in $p_{\text {buoy }}$ between the top and bottom of the thermal, $\left(\Delta p_{\text {buoy }}\right)_{\text {th }}=\rho_{0}\left(\alpha^{2} \bar{B} \Delta H / 2+\alpha^{2}\right)$, assuming spherical geometry of the region of positive buoyancy relative to the environment such that $H=2 R$ in the equation from Morrison (2016a). Here, $H$ and $R$ are the updraft height and radius, respectively, and $\alpha$ is the ratio of $w$ horizontally averaged across the updraft to $w$ at the updraft center. We also approximate the difference in $p_{\text {buoy }}$ between $H_{t}$ and $H_{m}$, denoted as $\Delta p_{\text {buoy }}$. We assume that the ratio $\Delta p_{\text {buoy }} /\left(\Delta p_{\text {buoy }}\right)_{\text {th }}$ scales with the fraction of the thermal's total vertically integrated buoyancy contained between $H_{m}$ and $H_{t}$, equal to $(1-y)$. Thus, $\Delta p_{\text {buoy }}$ is obtained from scaling $\left(\Delta p_{\text {buoy }}\right)_{\text {th }}$ by $(1-y)$ to yield

$$
\Delta p_{\text {buoy }} \approx \frac{\rho_{0}(1-y) \alpha^{2} \bar{B} \Delta H}{2+\alpha^{2}} .
$$

Note that there is some uncertainty with this approach because, in general for atmospheric moist thermals, there is nonzero buoyancy outside of the thermal boundaries, which influences the $p_{\text {buoy }}$ field within them. This is neglected for simplicity since, as shown later, $p_{\text {buoy }}$ has a limited impact on $\lambda$. Combining (11) and (8) gives

$$
\Delta p_{\text {buoy }} \approx \frac{\rho_{0}(1-y) \alpha^{2} w_{m}^{2}}{2 b\left(2+\alpha^{2}\right)} .
$$

It is assumed that the distribution of $p_{\text {dynam }}$ between $H_{m}$ and $H_{t}$ along the thermal's vertical axis of symmetry follows the scaling of $\Delta p$ as a function of $w_{m}^{*}$ and $\lambda$ from Hill's vortex given by (4). This is reasonable since LES studies have shown the overall flow structure of moist 
convective thermals is similar to Hill's vortex (Sherwood et al. 2013; Romps and Charn 2015; Hernandez-Deckers and Sherwood 2016; Moser and Lasher-Trapp 2017). Note that here we only use Hill's vortex scaling of $p_{\text {dynam }}$ between $H_{m}$ and $H_{t}$ and make no other assumptions about the $p_{\text {dynam }}$ field. Using $w_{m}=w_{m}^{*}-w_{t}^{*}=(1-\lambda) w_{m}^{*}$, squaring this expression, and combining it with (4) gives

$$
\Delta p_{\text {dynam }}=\frac{9}{8} \frac{\lambda^{2}}{(1-\lambda)^{2}} \rho_{0} w_{m}^{2} .
$$

Combining (12) and (13) yields

$\Delta p=\Delta p_{\text {dynam }}+\Delta p_{\text {buoy }} \approx\left[\frac{9 \lambda^{2}}{8(1-\lambda)^{2}}+\frac{(1-y) \alpha^{2}}{2 b\left(2+\alpha^{2}\right)}\right] \rho_{0} w_{m}^{2}$.

Next, we derive an expression for $b$ in terms of the vertically integrated buoyancy. For the simulated updrafts described in Peters (2016) and Morrison (2016b), the magnitude of $w_{m}^{*}$ was accurately diagnosed by vertically integrating the sum of $B$ and $-(1 / \rho)\left(\partial p_{\text {buoy }} / \partial z\right)$ (i.e., the effective buoyancy) over the region with $B>0$, with $p_{\text {dynam }}$ neglected. Thus, $p_{\text {dynam }}$ is important in explaining why $w_{m}^{*}$ occurs below the level of maximum vertically integrated buoyant forcing, but it does not appreciably impact the magnitude of $w_{m}^{*}$. This is because of the vertical structure of the $p_{\text {dynam }}$ field, with an upward-directed $p_{\text {dynam }}$ force in the lower part of the updraft approximately balanced by a downwarddirected $p_{\text {dynam }}$ force near the updraft top.

Given this finding, we approximate $w_{m}^{*}$ by vertically integrating the vertical momentum equation along $r=0$ over heights where $B>0$. For simplicity, it is assumed that the updraft region with $B>0$ is contained between the surface $(z=0)$ and the thermal top $\left(z=H_{t}\right)$, neglecting regions with $B<0$ between the surface and thermal top. This gives

$w_{m}^{* 2} \approx \int_{z=0}^{z=H_{t}} \frac{\partial\left(w^{* 2}\right)}{\partial z} d z=-\frac{2 \Delta p_{\text {buoy }, u}}{\rho_{0}}+2 \int_{z=0}^{z=H_{t}} B d z$,

where $\Delta p_{\text {buoy }, u} \equiv \int_{z=0}^{z=H_{t}}\left(\partial p_{\text {buoy }} / \partial z\right) d z$. Similar to previous derivations (Morrison 2016a; Peters 2016), (15) assumes that the vertical integral of buoyancy at time $t$ is equal to the integral of the Lagrangian derivative $d w^{*} / d t=w^{*} d w^{*} / d z$ over the path traveled by a parcel from the initial time to $t$ (i.e., $w^{*} \partial w^{*} / \partial z=w^{*} d w^{*} / d z$ ), implying $\partial w^{*} / \partial t=0$ along the parcel path. This approach gives a close correspondence between values of $w_{m}^{*}$ calculated using the simulated $B$ field and $w_{m}^{*}$ obtained directly from the simulations as shown in section 4.
The right-hand side of (15) is approximated following Morrison [2016a, their (27)]. We use $w_{m}^{*}=w_{N}$ from that paper, where $w_{N}$ is the maximum vertical velocity accounting for $B$ and $p_{\text {buoy }}$ but neglecting $p_{\text {dynam }}$. We also assume for simplicity that CAPE from (27) in Morrison (2016a) can be approximated as CAPE $\approx \int_{z=0}^{z=H_{t}} B d z$; this follows from the assumption above that the updraft region with $B>0$ is contained between $z=0$ and $z=H_{t}$, neglecting the impact of $B<0$ between these heights. With these approximations, after rearranging terms, (27) from Morrison (2016a) is written as

$$
w_{m}^{*_{2}} \approx \frac{2}{1+\frac{\alpha^{2} A_{r}^{2}}{2}} \int_{z=0}^{z=H_{t}} B d z
$$

where $A_{r} \equiv 2 R / H$ is the width-to-height aspect ratio of the updraft's region of positive buoyancy, relative to the environment, including that in the thermal head and wake regions (see Fig. 2).

Combining (16) and (8) with $w_{m}^{2}=\left(w_{m}^{*}-w_{t}^{*}\right)^{2}=$ $(1-\lambda)^{2} w_{m}^{* 2}$ and rearranging terms, $b$ can be expressed as

$$
b \approx \frac{(1-\lambda)^{2}}{\left(1+\frac{\alpha^{2} A_{r}^{2}}{2}\right)} \frac{\int_{z=0}^{z=H_{t}} B d z}{\bar{B} \Delta H}=\frac{(1-\lambda)^{2}}{\left(1+\frac{\alpha^{2} A_{r}^{2}}{2}\right)} h,
$$

where $h \equiv\left(\int_{z=0}^{z=H_{t}} B d z\right) /(\bar{B} \Delta H)$ is the ratio of the vertically integrated buoyancy from the surface to the thermal top and the vertically integrated buoyancy within the thermal. Generally, the magnitude of $h$ will be small (e.g., $h \sim 1$ ) when the thermal is in the lower troposphere and large (e.g., $h \gg 1$ ) when it is in the upper troposphere. This is because the ratio of the vertically integrated buoyancy within the thermal, relative to that in the stem below (Fig. 2), generally decreases as thermals rise through the troposphere.

Combining (8), (10), (14), and (17) yields a quadratic expression for $\lambda$ that depends only on $\alpha, A_{r}$, and the nondimensional buoyancy-dependent parameters $h$ and $y$. This is solved for $\lambda$ to give

$$
\lambda=\frac{-\beta+\sqrt{\beta^{2}-4 A C}}{2 A},
$$

where $A=5 / 4, \quad \beta=2$, and $C=(1-y)\left[1+\left(\alpha^{2} A_{r}^{2} / 2\right)\right]$ $\left\{\left[\alpha^{2} /\left(2+\alpha^{2}\right)\right]-1\right\} / h-1$. In (18), we have taken the positive root of the quadratic expression, which yields physical solutions. Since $w_{t}^{*}=\lambda w_{m}^{*}$, an estimate for the ascent rate of the thermal top follows from (16): 


$$
w_{t}^{*} \approx \lambda \sqrt{\frac{2}{1+\frac{\alpha^{2} A_{r}^{2}}{2}} \int_{z=H_{0}}^{z=H_{t}} B d z .}
$$

Values of $\lambda$ from (18) as a function of $y$ and $h$ are shown in Fig. 3. These calculations use $\alpha=0.78$, which is the mean value from the numerical simulations, and the scaling $A_{r}=2 / h$; see section 4 for details. As a sanity check, taking the limit $h \rightarrow \infty$ in (18), meaning that the thermal's buoyancy vanishes, returns the value of $\lambda=0.4$, consistent with Hill's vortex, which is nonbuoyant. Values of $\lambda$ decrease with increasing $h$, ranging from greater than 1 for $h \ll 1$ to less than 0.45 for $h>5$. Likewise, values of $\lambda$ decrease with increasing $y$, albeit by a smaller amount (up to $\sim 0.25$ ) over the range of $y$ from the simulations (approximately $0.05<y<0.75$ ). This implies that $\lambda$ is larger when the thermal's vertical distribution of positive buoyancy is concentrated near the thermal top.

As discussed in the introduction, earlier observational studies related $w_{t}^{*}$ to the buoyant forcing via an empirical Froude number $F_{r}$. For a spherical thermal with $B=0$ in the environment outside the thermal, meaning $h=1$ and $A_{r}=1$, we can express $F_{r}=2\left(1+\alpha^{2} / 2\right)^{-1 / 2} \lambda$ using (16) combined with $F_{r}=w_{t}^{*} /(\bar{B} R)^{1 / 2}$ from Scorer (1957). Assuming $\alpha=0.78$ as above and a homogeneous buoyancy field within the thermal with a maximum $w$ in the thermal center so that $y=0.5,(18)$ gives $\lambda=0.56$ and correspondingly $F_{r}=0.98$. While the thermal buoyancy structure was not quantified in the cloud observational and laboratory studies of Malkus and Scorer (1955) and Scorer (1957) so that a direct comparison is not possible, the theoretical $F_{r}=0.98$ is similar to the mean values of 0.89 (Malkus and Scorer 1955) and 1.2 (Scorer 1957) from these studies.

We also calculated theoretical values of $\lambda$ but with $\Delta p_{\text {buoy }}$ in (12) equal to zero and with an increase of $\Delta p_{\text {buoy }}$ by a factor of $2 ; \Delta p_{\text {buoy }}$ affects $\lambda$ since it counteracts part of the upward buoyant force in the upper part of the thermal, but the overall impact is small (Figs. 3b,c). When it is neglected, or scaled by a factor of 2 , the change in $\lambda$ ranges from $\sim 0.01$ for $\lambda=0.45$ to $\sim 0.1$ for $\lambda=1$. Thus, assumptions regarding $\Delta p_{\text {buoy }}$ are not very important for estimating $\lambda$.

This paper focuses on positively buoyant moist convective thermals. However, when $h$ is negative, that is, when the net buoyancy within the thermal is negative while the total buoyancy integrated from the surface to the thermal top is positive (e.g., overshooting convection), the expression for $\lambda$ gives values less than that for Hill's vortex of 0.4. This makes sense, since $h<0$ and $y>0$ implies negative buoyancy between $H_{m}$ and $H_{t}$, which contributes a downward-directed buoyant force

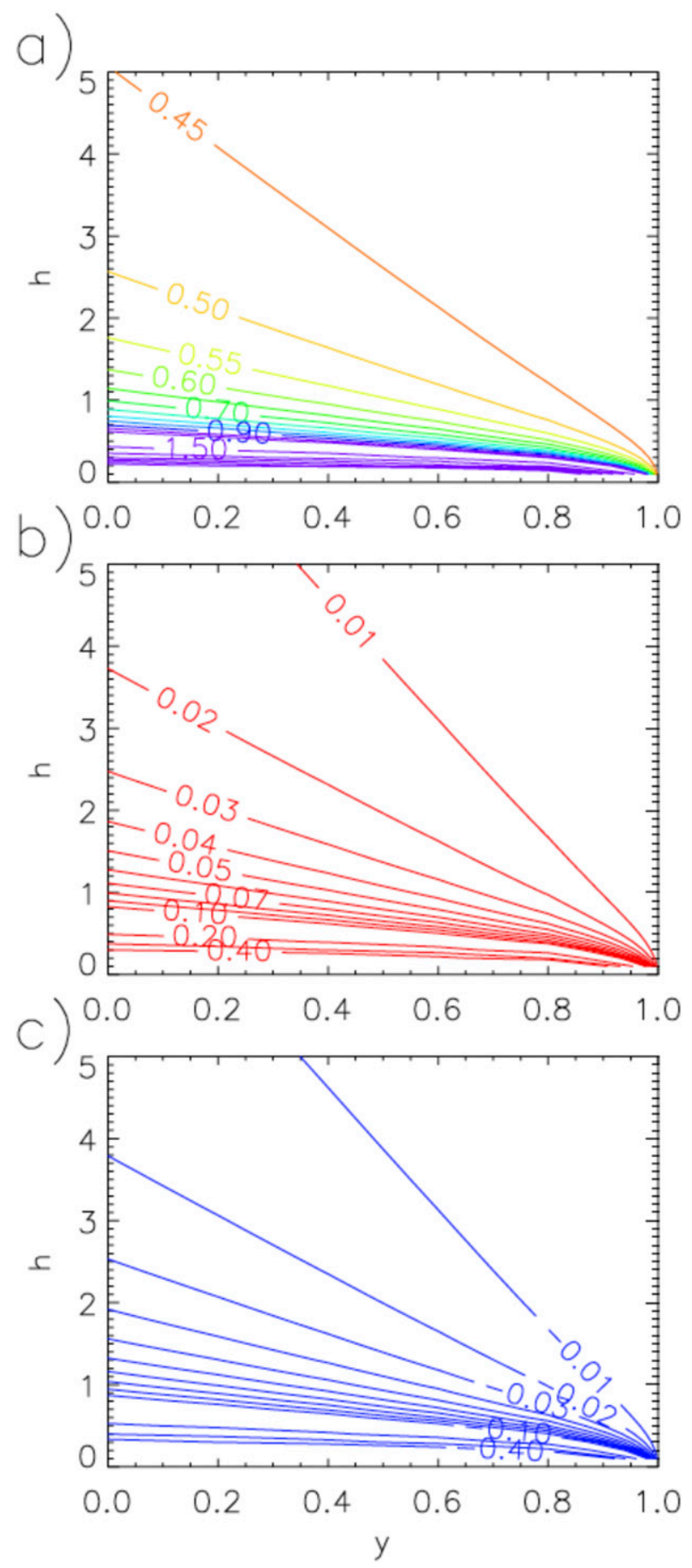

FIG. 3. (a) Theoretical values of $\lambda$ from (18) as a function of parameters $y$ and $h$. The contour lines in (a) have colors matching the $\lambda$ values shown in Fig. 9. (b) Difference in $\lambda$ from (18) with that neglecting $\Delta p_{\text {buoy }}$ within the thermal (red contour lines). (c) Difference in $\lambda$ from (18) with that increasing $\Delta p_{\text {buoy }}$ by a factor of 2 (blue contour lines). Contour intervals in (b) and (c) are every 0.01 between -0.1 and 0.1 and every 0.1 outside of this range. 
in addition to the downward-directed force from $-(1 / \rho)\left(\partial p_{\text {dynam }} / \partial z\right)$

An assessment of the theoretical expressions for $\lambda$ and $w_{t}^{*}$ by comparison with numerical simulations is presented in section 4 . In the next section, we describe the configuration of these numerical experiments.

\section{Description of the numerical model and setup}

The development and evolution of idealized moist deep convective thermals is simulated within an otherwise initially homogeneous environment. We use the Cloud Model 1 (CM1), version 18 (Bryan and Fritsch 2002), which is a fully compressible and nonhydrostatic model that was designed for simulating cloud processes. CM1 is configured in three dimensions with horizontal and vertical grid spacings of $100 \mathrm{~m}, 513$ grid points for each horizontal direction (equating to a $51.2-\mathrm{km}$-wide domain), and 201 grid points in the vertical (equating to a 20-km-deep domain). CM1 uses a time-splitting acoustic scheme, with the acoustic time step set to $1 / 6$ of the overall model time step of $0.5 \mathrm{~s}$. The simulation time is $15 \mathrm{~min}$. Lateral boundary conditions are periodic, and model top and bottom boundary conditions are free slip. Microphysics is represented by a modified version of the Morrison et al. (2005) double-moment scheme. All microphysical processes except cloud liquid water condensation and evaporation are turned off to avoid complications associated with precipitation formation and ice processes. Mixing from subgrid-scale eddies is parameterized following Smagorinsky (1963). The initial thermodynamic profiles follow from the analytic sounding of Weisman and Klemp (1982), which supports deep convection with a convective available potential energy of approximately $2700 \mathrm{~J} \mathrm{~kg}^{-1}$. Convection is initiated by adding a bubble with a maximum perturbation potential temperature of $2 \mathrm{~K}$ centered at a height of $1.5 \mathrm{~km}$. The initial bubble radius is varied in the simulations from 500 to $4000 \mathrm{~m}$. The environmental wind is set to zero. The distributions of $p_{\text {dynam }}$ and $p_{\text {buoy }}$ are obtained by solving the diagnostic pressure equation using a fast Fourier transform in the horizontal direction (which is consistent with periodic lateral boundary conditions) and a fast Fourier cosine transform in the vertical direction (which is consistent with Neumann top and bottom boundary conditions).

The simple idealized framework utilized here is intended to capture fundamental aspects of the scaling behavior of moist deep convective thermals. This framework facilitates a direct, quantitative comparison with the theoretical expressions since 1) the convective initiation and initial updraft properties are well controlled in all simulations; 2) updraft size scales with the initial bubble size, allowing systematic testing over a wide range of thermal sizes; and 3) flow irregularities and asymmetries are limited, and vertical axisymmetry around the thermal center is retained (the latter consistent with the geometry assumed by the theoretical derivation). High resolution (100-m horizontal and vertical grid spacings) allows the model to capture details of the thermal toroidal circulation and other aspects of the flow such as changes in thermal volume over time. However, explicitly resolved turbulence is limited in these simulations because of the finite time required to spin up grid-scale turbulent motion and develop an energy cascade (e.g., Chow et al. 2005; Lebo and Morrison 2015). Thus, we emphasize that these simulations are not LES as it is traditionally defined and should instead be considered as high-resolution simulations of diffusive moist thermals. As will be shown, though simple, this framework captures key features of moist convective thermals in LESs (Sherwood et al. 2013; Romps and Charn 2015; Hernandez-Deckers and Sherwood 2016; Moser and Lasher-Trapp 2017), including a flow structure similar to Hill's vortex and "stickiness" due to a significant fraction of the upward buoyant forcing that is countered by a downward-directed perturbation pressure gradient force. While the effects of explicitly resolving turbulent eddies on $\lambda$ and the thermal ascent rate are uncertain, LES studies have also shown that momentum mixing from turbulent eddies is relatively unimportant in the vertical momentum budget of thermals (Sherwood et al. 2013; Romps and Charn 2015; Hernandez-Deckers and Sherwood 2016).

The simulated thermals have radii that are roughly one-half the radius of the initial bubbles and thus range from about $300 \mathrm{~m}$ to $2 \mathrm{~km}$. Note that previous LES studies (e.g., Hernandez-Deckers and Sherwood 2016) have found most cumulus thermals to be small $(<300-\mathrm{m}$ radius). Thus, the thermals in our simulations are on the large end of the size range from LESs, but our intent is to focus on thermals that have a scale similar to the updraft structures themselves rather than those associated with smaller-scale turbulent eddies.

\section{Comparison of the numerical simulations and theory}

All of the simulations initiate moist convection within the first few minutes and subsequently produce cloud updrafts that ascend through most or all of the troposphere. The updraft structure consists of an ascending buoyant plume with a toroidal circulation in the thermal-like flow at the rising plume head (Figs. 4-6). This structure is similar to the starting plume described by Turner (1962). Thermal-like structures are also 

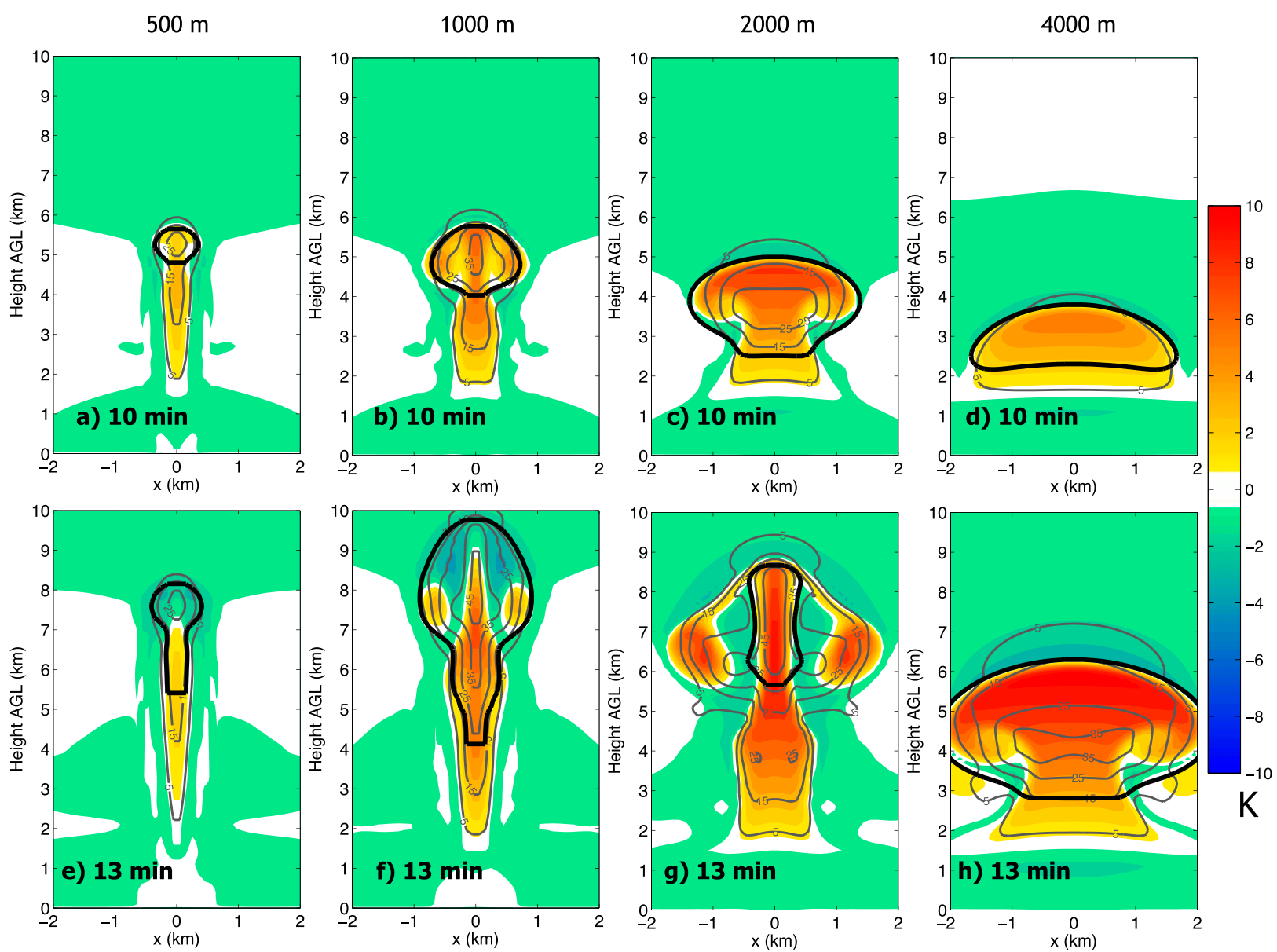

FIG. 4. Perturbation potential temperature $\theta^{\prime}$ (color shading; K), and $w^{*}$ (gray contour lines at intervals of $5 \mathrm{~m} \mathrm{~s}^{-1}$, starting at $5 \mathrm{~m} \mathrm{~s}{ }^{-1}$ ). The $x$ axis is the horizontal distance with zero located along the thermal's central axis. Thick black lines indicate the boundaries of the thermal at the updraft head, defined as described in section 4. (a),(e) The 500-m bubble run at 10 and 13 min. (b),(f) The 1000-m run at 10 and 13 min. (c),(g) The 2000-m run at 10 and 13 min. (d),(h) The 4000-m run at 10 and 13 min.

evident lower down in the wake region, particularly for the wider updrafts, but here, we focus on the thermals located at the updraft head. The boundaries of these thermals are indicated by the blue lines in Fig. 4, defined using a methodology similar to Romps and Charn (2015). The thermal structure located at the updraft head is found by moving downward from the model top along $r=0$ (defined by the thermal's vertical axis of symmetry at $x=0$ in Fig. 4) and locating the first local maximum in divergence with $w>1 \mathrm{~m} \mathrm{~s}^{-1}$, which is how the thermal top is defined. The vertical velocity at this level provides $w_{t}^{*}$, which is then used to calculate the streamfunction using $\psi(r, z) \equiv 2 \pi \int_{0}^{r} \rho\left(w^{*}-w_{t}^{*}\right) r^{\prime} d r^{\prime}$. Thermals are defined by regions with $\psi>0$. The lower thermal boundary is defined by the height where $w^{*}=w_{t}^{*}$ along the thermal's vertical axis since the streamfunction there is zero. Note that buoyancy and the buoyant perturbation pressure from the simulations are defined relative to the initial thermodynamic sounding, which is close to the horizontally averaged thermodynamic profiles. Both the 500- and 1000-m bubble simulations experience a large reduction in positive buoyancy by $13 \mathrm{~min}$, whereas the larger thermals retain considerable positive buoyancy at $13 \mathrm{~min}$.

Maximum vertical velocities generally occur $1-3 \mathrm{~km}$ below cloud top approximately at the thermal center (Fig. 4). The distributions of $p_{\text {buoy }}$ (Fig. 5) are broadly consistent with previous studies (Morrison 2016a; Peters 2016), with a local high in $p_{\text {buoy }}$ near the updraft top and a local low near the updraft bottom, although the structure of $p_{\text {buoy }}$ becomes increasingly complex over time as the buoyancy field evolves. The magnitude of $p_{\text {buoy }}$ is smaller for narrow compared to wide updrafts, consistent with the idea that narrow updrafts have a larger effective buoyancy (Jeevanjee and Romps 2016; Peters 2016; Jeevanjee 2017). The distribution of $p_{\text {dynam }}$ 

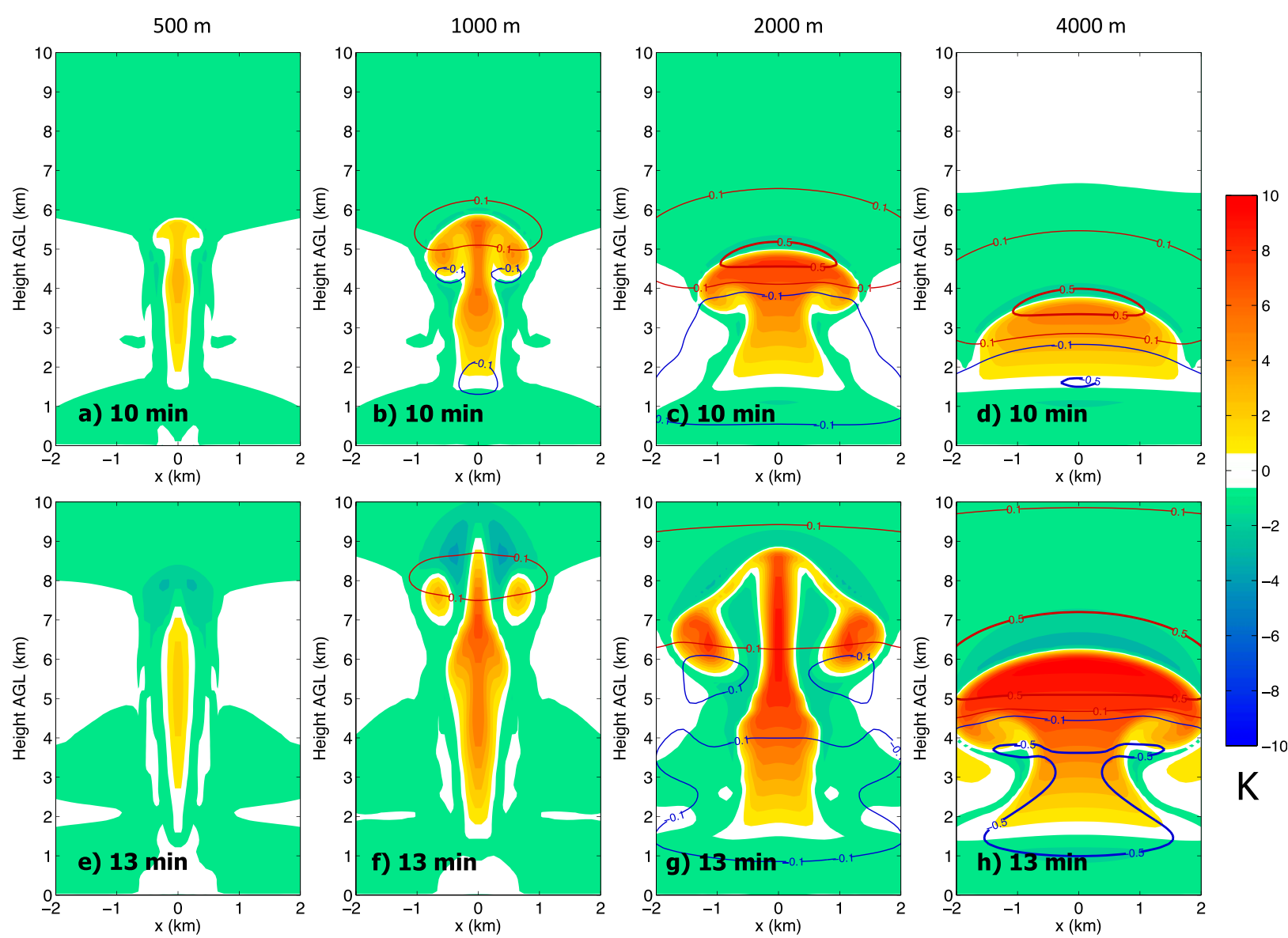

FIG. 5. As in Fig. 4, but for $p_{\text {buoy }}$, where red contour lines show $p_{\text {buoy }}>0$ starting at $0.1 \mathrm{hPa}$ and increasing with intervals of $0.5 \mathrm{hPa}$. Blue contour lines indicate $p_{\text {buoy }}<0$ starting at $-0.1 \mathrm{hPa}$ and decreasing with intervals of $0.5 \mathrm{hPa}$.

(Fig. 6) is characterized by low pressure associated with the thermal's toroidal circulation, high pressure near the updraft top, and high pressure near the updraft bottom. This is consistent with the conceptual model from Peters (2016) and broadly similar to the pressure distribution within Hill's vortex. As with $p_{\text {buoy }}$, the magnitude of $p_{\text {dynam }}$ is smaller for narrow updrafts compared to wide ones.

In subsequent analysis, we focus on time periods when the thermals/updrafts rise through the troposphere. These time periods are defined in each simulation as when 1) the thermal top is below $12 \mathrm{~km}$, the approximate tropopause height; 2 ) the thermal bottom, defined by the level below $H_{m}$ where the vertical velocity is equal to $w_{t}^{*}$, exceeds $1 \mathrm{~km}$ in height; and 3 ) the thermal top ascends at greater than $2 \mathrm{~m} \mathrm{~s}^{-1}$. The $\lambda$ from the simulations is calculated using a centered time difference and then smoothed using a Gaussian filter of radius $15 \mathrm{~min}$. Results are shown at 1-min intervals as the thermals rise.

The parameters $y$ and $h$ in the theoretical expressions are directly obtained from the simulations at each analysis time; $\alpha$ is derived from the simulations by defining the updraft region as that with $w \geq 1 \mathrm{~m} \mathrm{~s}^{-1}$ and perturbation potential temperature $\geq 1 \mathrm{~K}$. At each vertical level within the updraft, we take the ratio of the horizontal-averaged $w$ and the maximum $w$ and then average these values vertically. A mean value of $\alpha=0.78$ for all of the cases is used in the theoretical calculations. The width-to-height aspect ratio of the buoyant updraft region $A_{r}$ is difficult to quantify precisely from the simulations since the width of the buoyant region varies with height (i.e., the relatively wide thermal "head" vs the narrower "stem" below, seen in Fig. 4, particularly for the wider updrafts). For simplicity, we relate $A_{r}$ to the nondimensional heightdependent parameter $h$ from section 2, which can be expressed as $h=\overline{B_{u}} H_{t} /(\bar{B} \Delta H)$, where $\overline{B_{u}}$ and $H_{t}$ are the mean buoyancy and distance from the surface to the thermal top, respectively, and $\bar{B}$ and $\Delta H$ are the mean buoyancy within the thermal and distance from the thermal bottom to top. Since the updraft height increases much more than the width as updrafts grow 

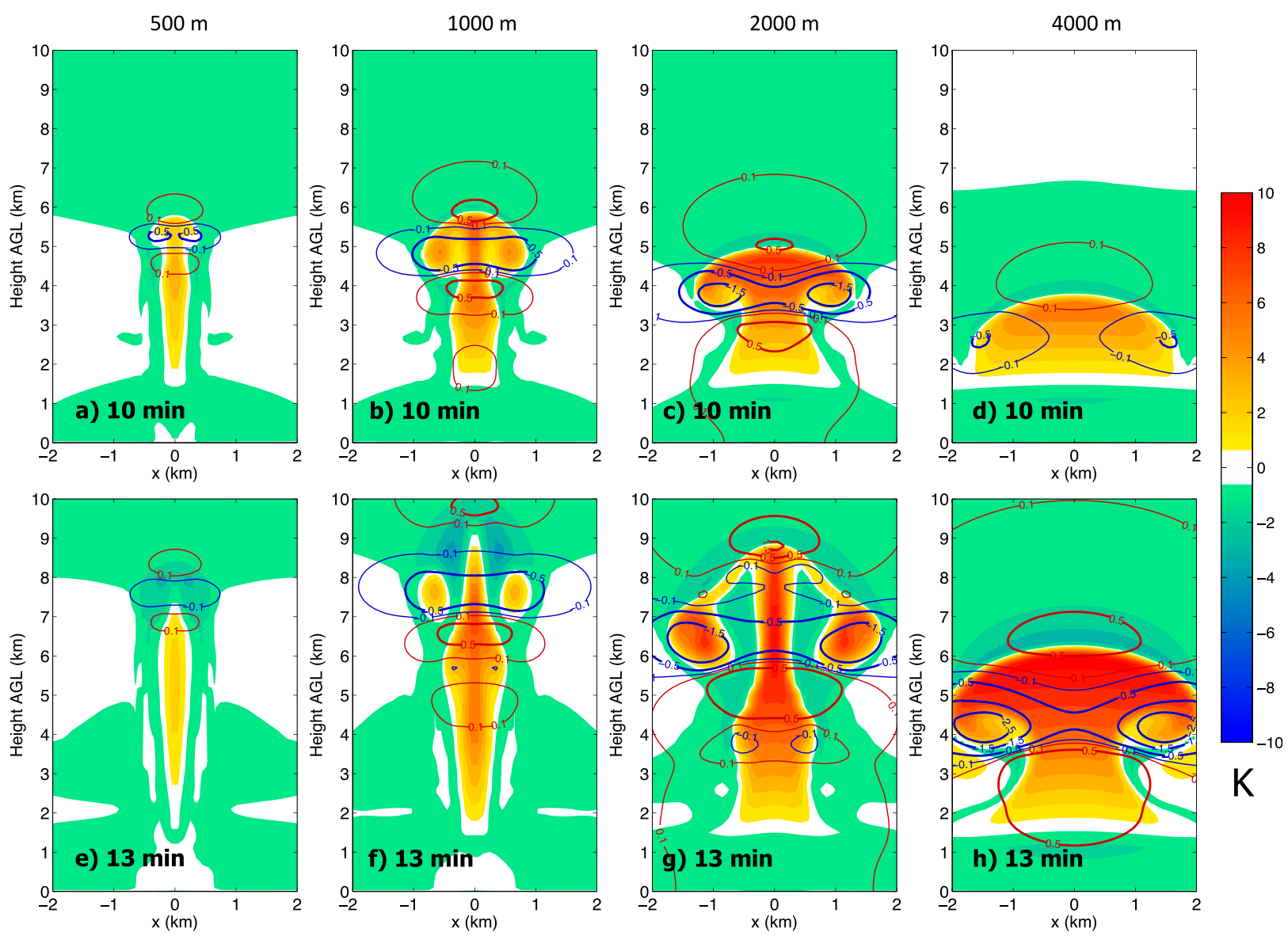

FIG. 6. As in Fig. 5, but with $p_{\text {dynam }}$ indicated by the contour lines.

(Fig. 4), it is reasonable to assume $A_{r}$ is proportional to $h^{-1}$. Here, we approximate $A_{r}=2 h^{-1}$, which gives a remarkably close correspondence between values of $w_{m}^{*}$ calculated using (16) and the simulated $w_{m}^{*}$ as shown below.

We first compare the theoretical representations of $\Delta p_{\text {dynam }}[(4)], \Delta p_{\text {buoy }}[(11)]$, and $w_{m}^{*}[(16)]$ to values of these quantities obtained directly from the simulations. To evaluate the theoretical expressions, linear correlation coefficients $r_{\mathrm{cc}}$ and normalized root-mean-square error NERR $\equiv \sqrt{(1 / N) \sum\left(a_{i}-b_{i}\right)^{2}} / \operatorname{range}\left(a_{i}\right)$ are computed, where $a_{i}$ is the value of a quantity directly from the model output, $b_{i}$ is the theoretical value of that quantity at the same time, $N$ is the number of data points, and "range" is the range of simulated values. When both $r_{\mathrm{cc}}$ and NERR are small, this implies large scatter but limited bias, while when both NERR and $r_{\mathrm{cc}}$ are large, there is a large bias but limited scatter.

Theoretical values of $\Delta p_{\text {dynam }}$ correspond well to simulated values (Fig. 7a) with $r_{\mathrm{cc}}=0.91$ and NERR $=0.49$. The comparison is somewhat poorer later in the simulations, especially for the 2000- and 4000-m initial bubble cases, with up to a factor of 2-2.5 overestimation by the theoretical values. This probably reflects the increased complexity of the flow field and hence larger deviations from Hill's vortex over time. There is a close correspondence between the simulated and theoretical $\Delta p_{\text {buoy }}$ for the $500-\mathrm{m}$ bubble simulations but large differences for the 1000-4000-m simulations (Fig. 7b). The theoretical and simulated $w_{m}^{*}$ are remarkably similar to one another, with $r_{\mathrm{cc}}>0.98$ and NERR $\leq 0.08$ (Fig. 8a).

The simulated and theoretical $\lambda$ have a reasonable correspondence for all bubble sizes and times, with $r_{\mathrm{cc}}=0.86$ and NERR $=0.15$ (Fig. 8b). While there is scatter, the theoretical and simulated $\lambda$ values also exhibit similar dependencies on $y$ and $h$ (Fig. 9), with an increase of $\lambda$ with a decrease of $h$ or $y$. For example, all simulated points with $\lambda>0.7$ occur when $h<1$, while all points with $\lambda<0.5$ have $h>1.2$. Values of $\lambda$ are generally largest early in the lifetimes of the thermals and decrease to about $0.4-0.5$ later. This behavior is explained by values of $h$ and $y$ that increase over time during the simulations as the thermals rise, because of 

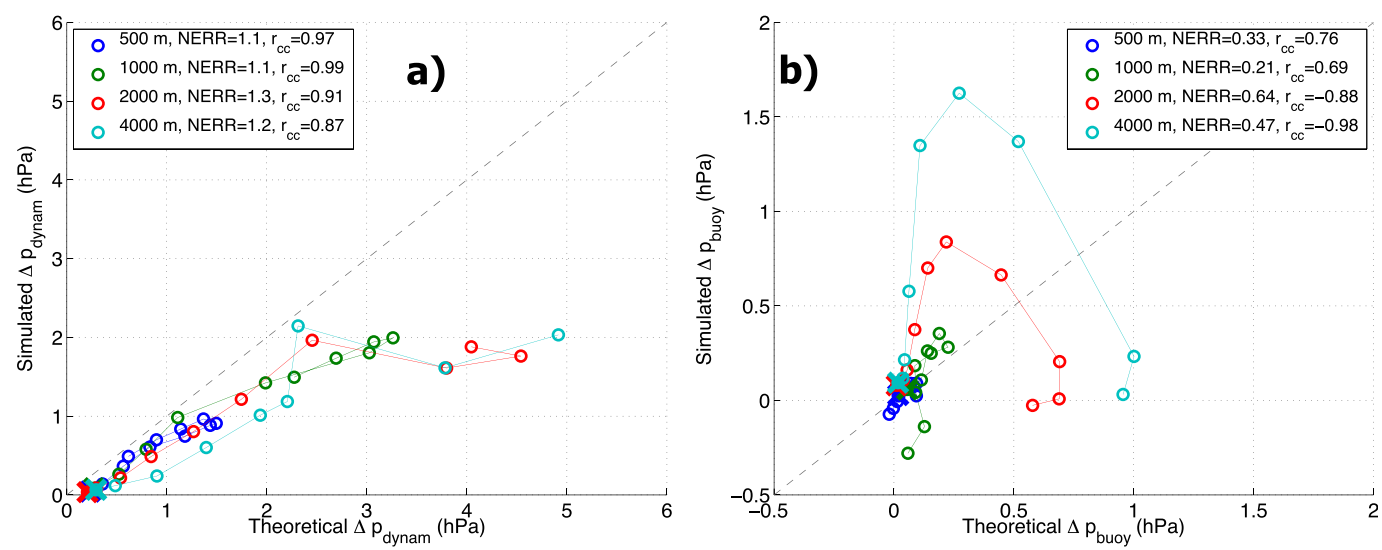

FIG. 7. Scatterplots (circles; blue: 500-m bubble simulation; green: 1000-m simulation; red: 2000-m simulation; cyan: 4000-m simulation) comparing theoretical and simulated quantities from 1-min model output, where lines connect temporally adjacent points and colored $\times$ marks indicate the earliest analysis time. Note that the $\times$ marks from the different simulations lie close to one another and are difficult to distinguish. (a) Plot of $\Delta p_{\text {dynam }}$ diagnosed by (13) with $w_{m}^{2}$ determined from the simulated thermals ( $x$ axis) vs $\Delta p_{\text {dynam }}$ directly obtained from the simulated thermals ( $y$ axis). (b) Plot of $\Delta p_{\text {buoy }}$ diagnosed by (12) with $w_{m}^{2}$ determined from the simulated thermals $(x$ axis) vs $\Delta p_{\text {buoy }}$ directly obtained from the simulated thermals ( $y$ axis). The NERR and $r_{\mathrm{cc}}$ are the normalized root-meansquare error and correlation coefficient for individual cases, respectively.

the generation of positive buoyancy in the stem region below the thermals and the reduction of buoyancy in the upper part of the thermals. This behavior is consistent with the laboratory thermal study of Sanchez et al. (1989). A direct comparison of the theoretical expressions with their results is not possible since they did not report buoyancy distributions within the thermals and in their environment. Nonetheless, they showed a clear decrease in the ratio of $w_{t}^{*}$ and $v_{s}$ (see the introduction), analogous to a decrease in $\lambda$, as thermals traveled to distances of several times their initial diameter (see their Fig. 8).

The buoyant perturbation pressure difference $\Delta p_{\text {buoy }}$ from $H_{t}$ to $H_{m}$ is generally smaller than $\Delta p_{\text {dynam }}$ except for a brief period during the 2000- and 4000-m bubble simulations when they are similar in magnitude. This implies that $\Delta p_{\text {dynam }}$ plays a comparatively larger role in determining $\lambda$ and also explains why the theoretical values of $\lambda$ correspond well with the simulated values despite fairly large differences between the theoretical and simulated $\Delta p_{\text {buoy. }}$. Given the close correspondence between the simulated and theoretical $w_{m}^{*}$ and the reasonable correspondence for $\lambda$, the theoretical thermal-top ascent rate $w_{t}^{*}=\lambda w_{m}^{*}$ matches well with the simulated values ( $r_{\mathrm{cc}} \geq 0.98$ for all cases).

To investigate further the roles of $p_{\text {dynam }}, p_{\text {buoy }}$, and $B$ in determining $\lambda$, we analyze back trajectories (Fig. 10) released from near the thermal top at $r=0$, defined along the vertical axis of symmetry around the thermal/ updraft center. Given this axisymmetry, horizontal velocity along these trajectories is zero, and hence, the trajectories are oriented in the vertical. Note that at $r=0$, the thermal top and bottom lie at the intersection of the streamlines and thus are local, instantaneous stagnation points with zero velocity relative to the ascent rate of the thermal top and bottom. Back trajectories released at 12 min near the thermal top show that parcels approximately follow the thermal bottom until about $10 \mathrm{~min}$ and then ascend through the depth of the thermal between 10 and $12 \mathrm{~min}$ (Figs. 10a,b). The sum of $-(1 / \rho)\left(\partial p_{\text {dynam }} / \partial z\right),-(1 / \rho)\left(\partial p_{\text {buoy }} / \partial z\right)$, and $B$ corresponds closely to the actual $d w^{*} / d t$ (cf. the black and gray dashed lines in Figs. 10c,d). This indicates the limited role of momentum entrainment and mixing on $w$ along $r=0$. This is expected since grid-scale turbulence is limited in this framework but nonetheless is consistent with LES studies showing the limited impact of entrainment and detrainment on the updraft momentum budget (Dawe and Austin 2011; de Roode et al. 2012; Sherwood et al. 2013; Romps and Charn 2015; Hernandez-Deckers and Sherwood 2016).

To understand the forces controlling $\lambda$, of particular interest are the forces that cause a decrease in $w$ between the level of maximum $w, H_{m}$, and the thermal top. Whereas $B$ contributes to increasing $w$ with time, $-(1 / \rho)\left(\partial p_{\text {buoy }} / \partial z\right)$ has little impact; $-(1 / \rho)\left(\partial p_{\text {dynam }} / \partial z\right)$ imparts a net upward force on $w$ between the thermal bottom and $H_{m}$ and a larger net downward forcing between $H_{m}$ and the thermal top. The latter leads to a reduction in $w$ of about $25 \mathrm{~m} \mathrm{~s}^{-1}$ between $H_{m}$ and the thermal top for the 1000-m bubble simulation and about $20 \mathrm{~m} \mathrm{~s}^{-1}$ for the $2000-\mathrm{m}$ simulation (Figs. 10e,f). Overall, 

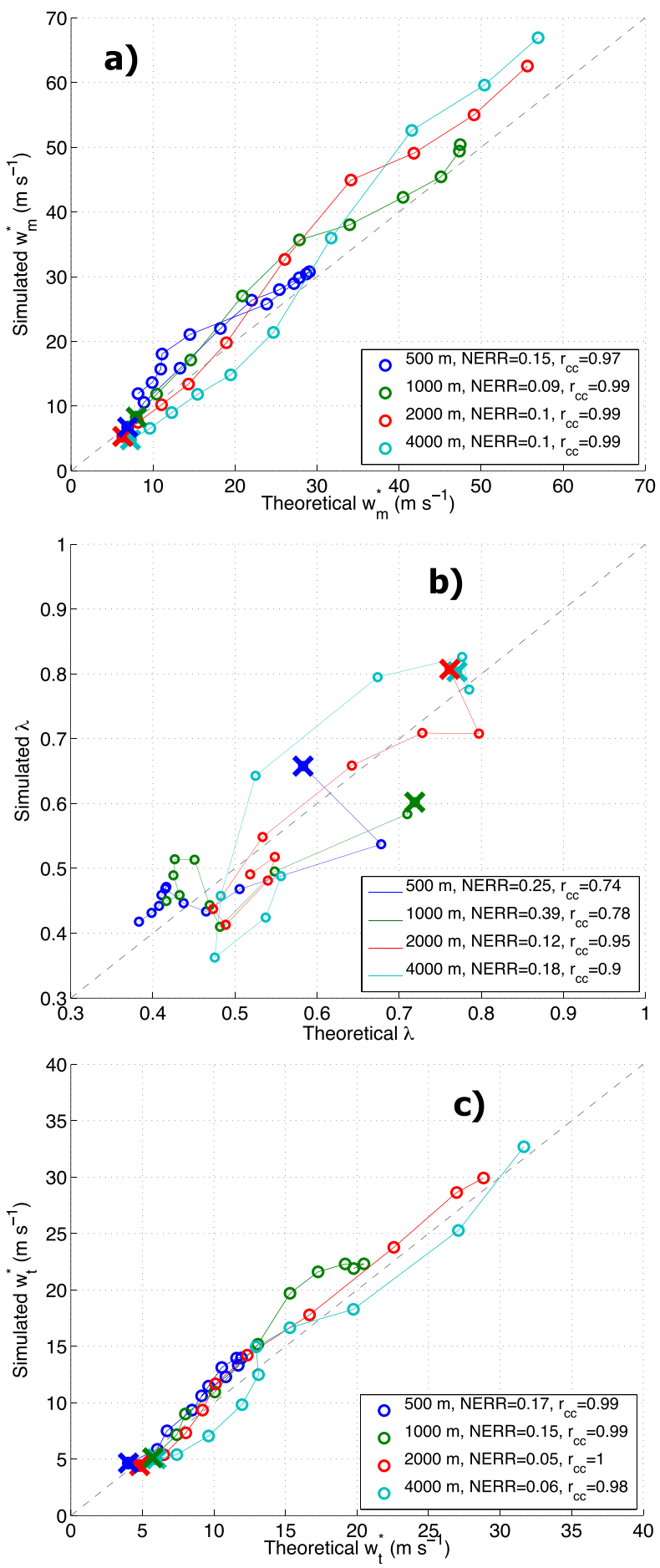

FIG. 8. As in Fig. 7, but for (a) plot of $w_{m}^{*}$ diagnosed by (16) ( $x$ axis) vs $w_{m}^{*}$ directly obtained from the simulated thermals ( $y$ axis). (b) Plot of $\lambda$ diagnosed by (18) with $y$ and $h$ obtained directly from model output ( $x$ axis) vs $\lambda$ directly obtained from the simulated thermals ( $y$ axis). (c) Plot of $w_{t}^{*}$ diagnosed by (19) ( $x$ axis) vs $w_{t}^{*}$ directly obtained from the simulated thermals ( $y$ axis).

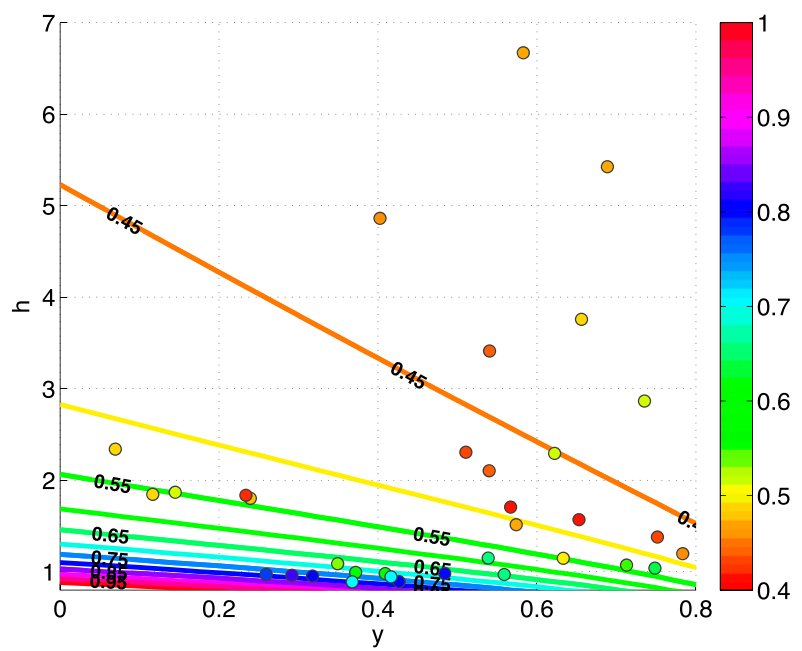

FIG. 9. Theoretical values of $\lambda$ as a function of $h$ and $y$ computed from (18) shown by color contour lines (corresponding to the color bar on the right) at intervals of 0.05 . Circle locations indicate values of $y$ and $h$ computed from 1-min snapshots of thermals simulated by CM1, and the circle color (which corresponds to the color bar on the right) indicates the simulated $\lambda$ value at that time.

these results highlight the competing effects of $B$ and $-(1 / \rho)\left(\partial p_{\text {dynam }} / \partial z\right)$ on $w$ between $H_{m}$ and the thermal top and hence their competing roles in controlling $\lambda$.

\section{Relationship to previous studies on thermal drag}

Sherwood et al. (2013), Romps and Charn (2015), and Hernandez-Deckers and Sherwood (2016) investigated the $w$ momentum budget of thermals in LESs of moist deep convection in the context of buoyant and drag forces. Although Sherwood et al. (2013) did not precisely define drag, their narrative implies that they interpreted drag as a force imparted by momentum mixing. The limited importance of momentum mixing in their simulations led them to conclude that moist convective thermals are minimally affected by drag and are thus "slippery." They suggested that this lack of drag was because the internal thermal flow structure was similar to Hill's vortex, which formally has no drag because its dynamic pressure field is vertically symmetric about the central vortex axis, and hence does not impart a net force on the vortex, and because its flow is inviscid.

In contrast to Sherwood et al. (2013), Romps and Charn (2015) explicitly defined drag as the net perturbation pressure gradient force acting on a thermal and calculated it directly from the pressure field in an LES of scattered deep convection. When defined in this way, the dominant balance within the $w$ momentum budget was between buoyancy and drag (i.e., "sticky" thermals). They found that the drag defined by the perturbation 
(a) $1000 \mathrm{~m}$ bubble trajectory Path

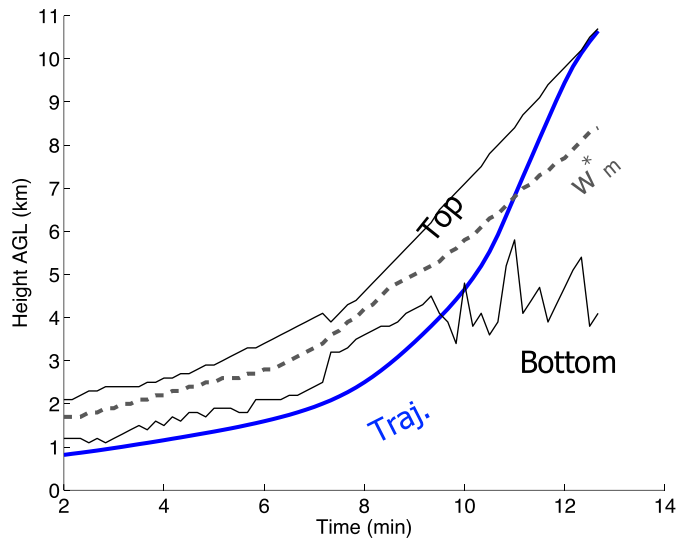

(c) $1000 \mathrm{~m}$ bubble at $12 \mathrm{~min}: \mathbf{d w} / \mathbf{d t}$

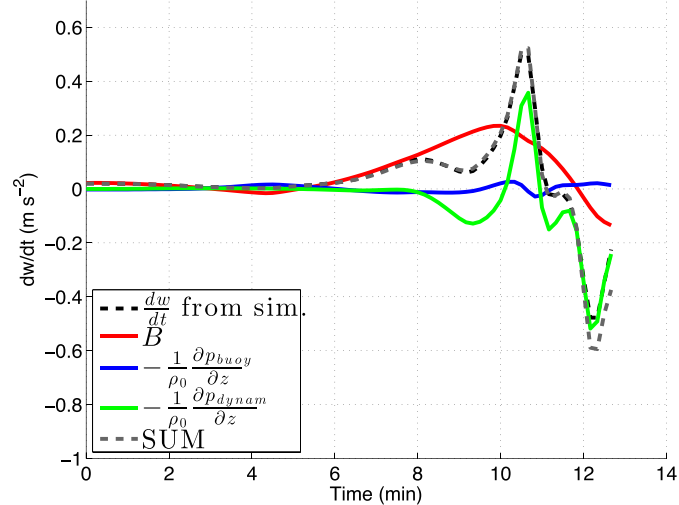

(e) $1000 \mathrm{~m}$ bubble at $12 \mathrm{~min}: \mathbf{w}$

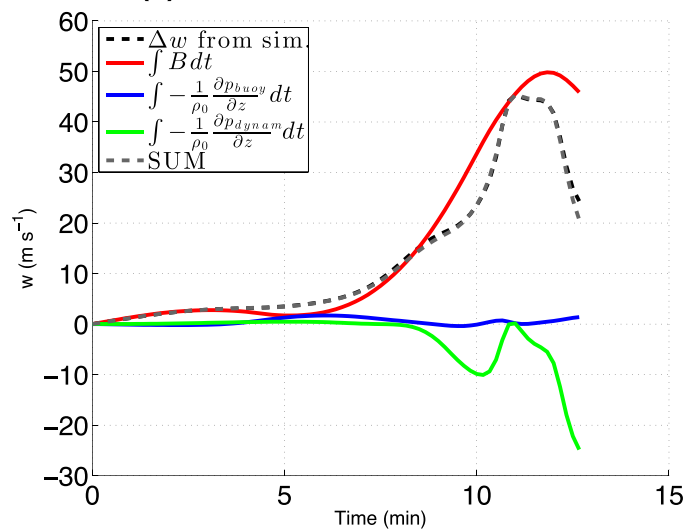

(b) $2000 \mathrm{~m}$ bubble trajectory Path

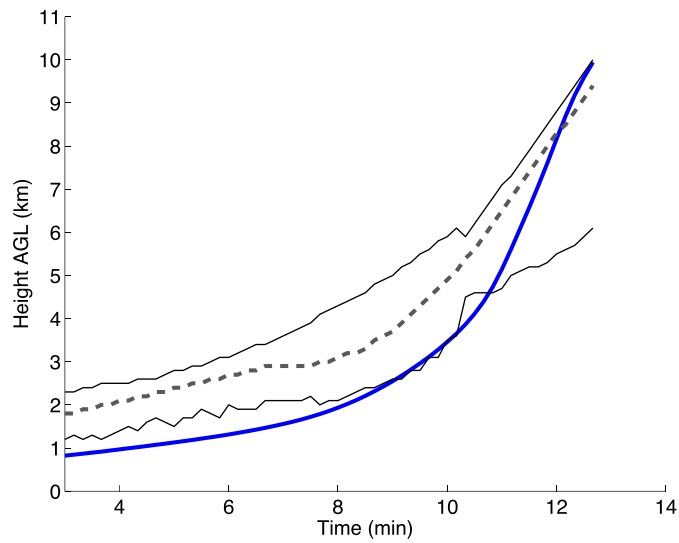

(d) $2000 \mathrm{~m}$ bubble at $12 \mathrm{~min}: \mathbf{d w} / \mathbf{d t}$

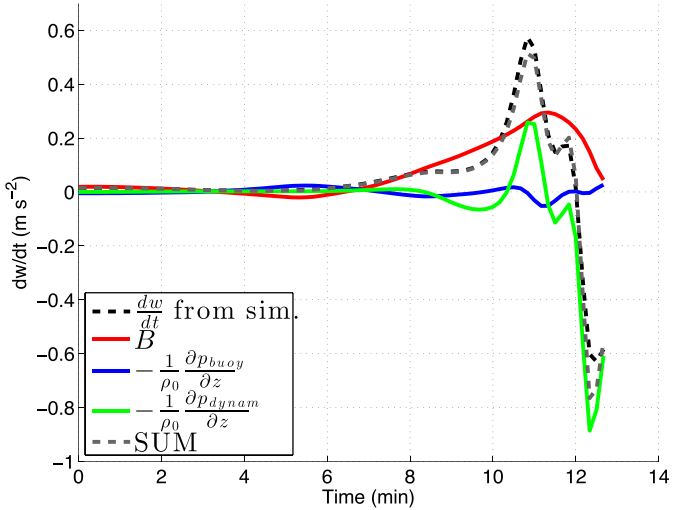

(f) $2000 \mathrm{~m}$ bubble at $12 \mathrm{~min}: \mathbf{w}$

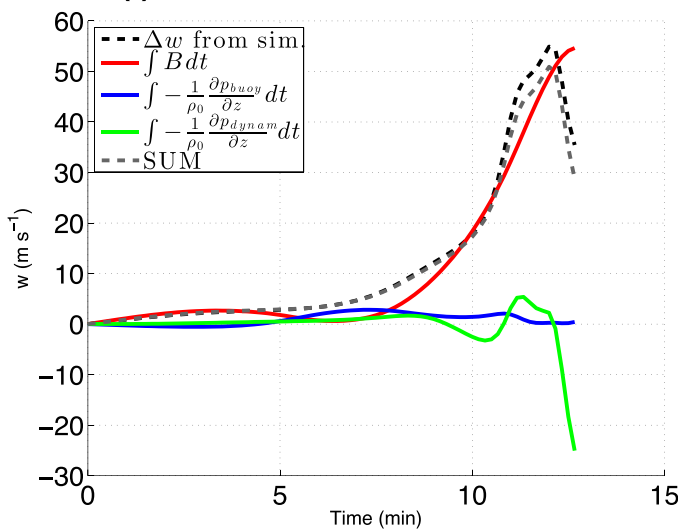

FIG. 10. Time series of quantities from simulations calculated along back trajectories released near the thermal top at $12 \mathrm{~min}$. (a) The trajectory height (blue line), the thermal top and bottom (black lines), and the height of maximum $w$ (gray dashed line) in the 1000-m bubble simulation. (b) As in (a), but for the 2000-m bubble simulation. (c),(d) Vertical accelerations $\left(\mathrm{m} \mathrm{s}^{-2}\right)$ are shown: $d w^{*} / d t$ computed directly from the modeled $w^{*}$ (black dashed line), $B$ (red line), $-(1 / \rho)\left(\partial p_{\text {buoy }} / \partial z\right)$ (blue line $),-(1 / \rho)\left(\partial p_{\text {dynam }} / \partial z\right)($ green line $)$, and $\mathrm{SUM} \equiv B-(1 / \rho)\left(\partial p_{\text {buoy }} / \partial z\right)-(1 / \rho)\left(\partial p_{\text {dynam }} / \partial z\right)$ (gray dashed line). (e),(f) Time integrals of the quantities in (c) and (d), which give the net change in $w^{*}\left(\mathrm{~m} \mathrm{~s}^{-1}\right)$ from a particular forcing. Trajectories were released along the thermal's vertical axis of symmetry and computed from 10 -s model output. Note that the black and gray dashed lines in (c)-(f) are very close to one another and difficult to distinguish. 
pressure gradient force was well correlated with the standard drag law for a sphere with a drag coefficient $C_{D} \approx 0.6$, larger than the $C_{D} \approx 0.2$ for high Reynolds number flow past a solid sphere. While seemingly contradicting the Sherwood et al. (2013) slippery thermal hypothesis, these differences are explained in part simply by differences in how these studies defined and interpreted drag, as was acknowledged by Romps and Charn (2015) and HernandezDeckers and Sherwood (2016).

In the rest of this section, we discuss how $\lambda$ and $w_{t}^{*}$ are connected to thermal drag and discuss results in the context of these previous studies. We first write the inviscid, Boussinesq vertical momentum equation as a Lagrangian derivative $d w^{*} / d t=B_{\text {eff }}-\rho_{0}^{-1} \partial p_{\text {dynam }} / \partial z$, where $B_{\text {eff }}$ is the effective buoyancy. Using the chain rule $d / d t=(d z / d t)(d / d z)=w^{*}(d / d z)$ and neglecting $p_{\text {dynam }}$ as discussed in section $2 b$, this expression well describes the vertical variation of the maximum $w^{*}\left(w_{m}^{*}\right)$ within a thermal ascending along the vertical axis of symmetry $(r=0)$. This implies $w_{m}^{*} d w_{m}^{*} / d z \approx B_{\text {eff }}$, where $B_{\text {eff }}$ is the effective buoyancy along $r=0$. Since $w_{t}^{*}=\lambda w_{m}^{*}$, we can write

$$
w_{t}^{*} \frac{d w_{t}^{*}}{d z} \approx \lambda^{2} B_{\mathrm{eff}}=\widehat{B_{\mathrm{eff}}}-\widehat{B_{\mathrm{eff}}}\left(1-\frac{\lambda^{2} B_{\mathrm{eff}}}{\widehat{B_{\mathrm{eff}}}}\right),
$$

where $\widehat{B_{\text {eff }}}$ is the thermal volume-averaged effective buoyancy, and it is assumed $d \lambda / d z=0$ for simplicity and tractability. While there is some vertical variation of $\lambda$ as shown in section 4 , the variation of $\lambda$ relative to its magnitude is much less than the variation of $w_{t}^{*}$ relative to its magnitude. Equation (20) is written in the form on the right-hand side based solely on the mathematical identity $x^{2} y=z-z\left(1-x^{2} y / z\right)$ that holds for any $x, y$, and $z \neq 0$. Integrating (20) over the path of the thermal as it ascends a distance $H$, assuming the initial thermal velocity is zero, gives $w_{t}^{* 2} \approx 2 \lambda^{2} \overline{B_{\text {eff }}} H$, where $\overline{B_{\text {eff }}}$ is the average effective buoyancy over $H$. This is combined with (20) to give

$$
w_{t}^{*} \frac{d w_{t}^{*}}{d z} \approx \widehat{B_{\text {eff }}}-\frac{\widehat{B_{\text {eff }}}}{2 H \lambda^{2} \overline{B_{\text {eff }}}}\left(1-\frac{\lambda^{2} B_{\text {eff }}}{\widehat{B_{\text {eff }}}}\right) w_{t}^{* 2}=\widehat{B_{\text {eff }}}-\widehat{F_{D}},
$$

where $\widehat{F_{D}}$ is a volume-average drag force on the thermal. Approximating $B_{\text {eff }}$ by its average value (i.e., $B_{\text {eff }} \approx \overline{B_{\text {eff }}}$ ), (21) is simplified to

$w_{t}^{*} \frac{d w_{t}^{*}}{d z} \approx \widehat{B_{\mathrm{eff}}}-\frac{1}{2 H}\left(\frac{B_{r}}{\lambda^{2}}-1\right) w_{t}^{* 2}=\widehat{B_{\mathrm{eff}}}-\widehat{F_{D}}$,

where $B_{r} \equiv \widehat{B_{\text {eff }}} / \overline{B_{\text {eff }}}$. If the ascent rate of the thermal top is equal to the volume-averaged thermal ascent rate, $w_{t}^{*}=\widehat{w^{*}}$ and (22) is equivalent to the thermal volumeaveraged $w$ momentum equation given by (24) below assuming Boussinesq flow and neglecting momentum mixing but written such that the $p_{\text {buoy }}$ force (or analogously the virtual mass force) is included in $\widehat{B_{\text {eff }}}$ and $\widehat{F_{D}}=\rho_{0}^{-1} \overline{\partial p_{\text {dynam }} / \partial z}$. Combining (22) with the standard drag law for a sphere $\widehat{F_{D}}=3 C_{D} w_{t}^{* 2} /(8 R)$, where $R$ is the thermal radius and $C_{D}$ is the drag coefficient, we can solve for $C_{D}$ :

$$
C_{D}=\frac{4 R}{3 H}\left(\frac{B_{r}}{\lambda^{2}}-1\right)
$$

This expression relates $C_{D}$ and $\lambda$. Since $\lambda$ is closely linked to the buoyancy field via parameters $y$ and $h$ primarily through vertical gradients of $p_{\text {dynam }}$ (as opposed to $p_{\text {buoy }}$ ) as shown in section $2 \mathrm{~b}$, this suggests that there is a drag on thermals associated primarily with $p_{\text {dynam }}$ due to flow asymmetries induced by the thermal buoyancy itself. Equation (23) shows that $C_{D}$ should increase as $\lambda$ decreases, for a given $B_{r}$, since a small value of $\lambda$ implies a slow ascent rate, which requires large drag. On the other hand, $C_{D}$ decreases as $B_{r}$ decreases. This makes physical sense, since small $B_{r}$ means that the thermal volume-averaged buoyancy is small compared with the buoyancy along the thermal's vertical central axis, and hence, less drag is needed to oppose the thermal volume-averaged buoyant force for a given $w_{t}^{*}$.

The simulations indicate a value of $B_{r} \sim 0.5$ (not shown). Combined with values of $\lambda \sim 0.4-0.8$, this gives a drag that counters up to $70 \%$ of the thermalaveraged buoyant forcing based on (20), which may help to explain why thermals tend to be sticky. Using $B_{r}=0.5$ and values of $R / H=0.5$ and $\lambda=0.65$ consistent with the simulated lower-tropospheric thermals in (23) gives $C_{D}=0.12 ; R / H=0.25$ and $\lambda=0.5$ for midtropospheric thermals gives $C_{D}=0.33 ; R / H=0.1$ and $\lambda=0.4$ for upper-tropospheric thermals gives $C_{D}=0.28$. This is reasonably in line with values of $C_{D}$ for convective thermals diagnosed from the LESs of Romps and Charn (2015) and Hernandez-Deckers and Sherwood (2016) of $\sim 0.6$ and $\sim 0-0.5$, respectively. Interestingly, the theoretical values of $C_{D}$ from (23) approach zero and even become negative as $\lambda$ increases to values larger than about 0.7 , for $B_{r} \approx 0.5$. Since large values of $\lambda(>0.65)$ occur early in the simulated thermal lifetimes (Fig. 8b), this suggests that small or negative $C_{D}$ should occur at low levels with an increase of $C_{D}$ to $\sim 0.3$ as thermals ascend and $\lambda$ decreases. This behavior is consistent with the profiles of $C_{D}$ diagnosed by Hernandez-Deckers and Sherwood (2016; see their Fig. 15). 
(a) $1000 \mathrm{~m}$ bubble

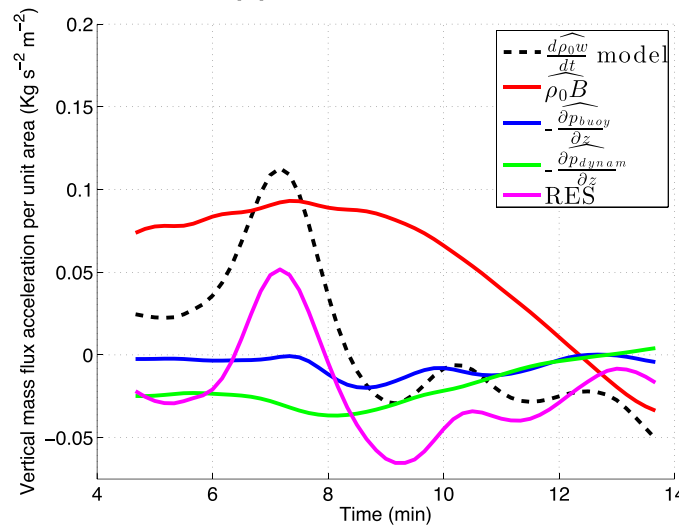

(c) $1000 \mathrm{~m}$ bubble

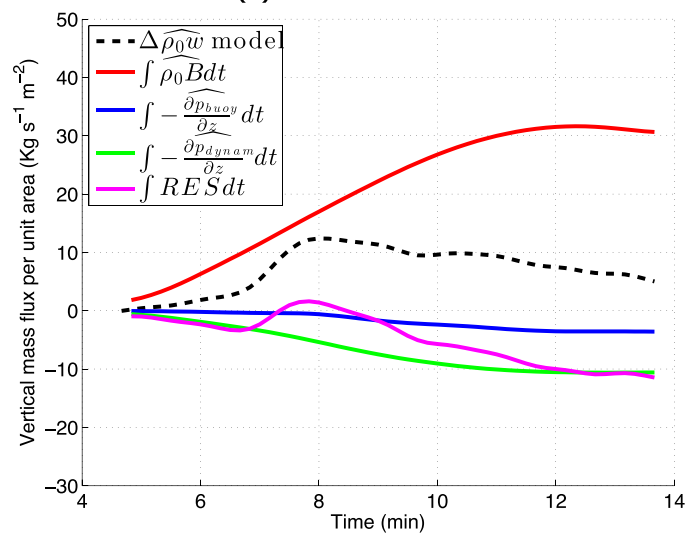

(b) $2000 \mathrm{~m}$ bubble

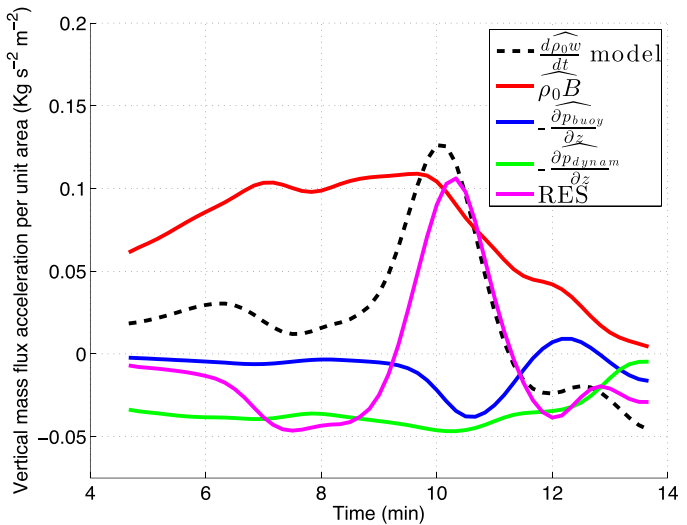

(d) $2000 \mathrm{~m}$ bubble

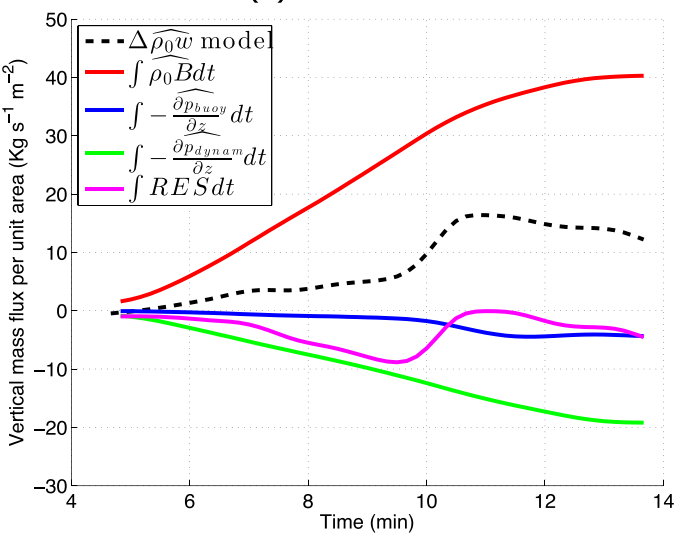

FIG. 11. (a) Time series of terms in the thermal volume-averaged vertical moment budget [(24)] from the 1000-m bubble simulation $\left(\mathrm{kg} \mathrm{s}^{-2} \mathrm{~m}^{-2}\right): \widehat{\rho B}$ (term A; red line), $-\overline{\partial p_{\text {buoy }} / \partial z}$ (term B; blue line), $-\overline{\partial p_{\text {dynam }} / \partial z}$ (term C; green line), RES (term D; magenta line), and ( $d / d t) \widehat{\rho w^{*}}$ (black dashed line) computed directly from the simulation with the time derivative estimated as a second-order centered difference. (b) As in (a), but from the 2000-m bubble simulation. (c),(d) Time integrals of the quantities in (a) and (b), which give the net change in $\rho w^{*}\left(\mathrm{~kg} \mathrm{~s}^{-1} \mathrm{~m}^{-2}\right)$ from a particular forcing.

To investigate forces impacting the simulated thermals, we compute the vertical momentum budget averaged over the thermal volume, written as

$$
\frac{d \widehat{\rho w^{*}}}{d t}=\underbrace{\widehat{\rho B}}_{\mathrm{A}}-\underbrace{\frac{\widehat{\partial p_{\text {buoy }}}}{\partial z}}_{\mathrm{B}}-\underbrace{\frac{\widehat{\partial p_{\mathrm{dynam}}}}{\partial z}}_{\mathrm{C}}-\underbrace{\mathrm{RES}}_{\mathrm{D}},
$$

where the overhats denote a volume average, term $\mathrm{A}$ is buoyancy, term $\mathrm{B}$ is the buoyant perturbation pressure forcing, term $\mathrm{C}$ is dynamic pressure forcing, and term $\mathrm{D}$ is a residual that implicitly includes the effects of momentum entrainment and detrainment [e.g., Romps and Charn 2015, their (3)]. The thermal boundaries are defined as described in section 4.

Figure 11 shows time series of the individual terms in (24) evaluated for the 1000- and 2000-m bubble simulations. Buoyancy provides a net upward force through the first $12 \mathrm{~min}$ of the simulations. In general, $-\overline{\partial p_{\text {dynam }} / \partial z}$ imparts a much larger net downward force on thermals than $-\overline{\partial p_{\text {buoy }} / \partial z}$, particularly early in the thermal lifetime. Composites of vertical profiles through the center of the thermal confirm the pattern of a net upward force from $B$, a small net downward force from $-\partial p_{\text {buoy }} / \partial z$, and a larger net downward force from $-\partial p_{\text {dynam }} / \partial z$ (Fig. 12). A net downward force from $-\partial \overline{p_{\text {dynam }} / \partial z}$ occurs because the downward-directed force in the upper part of the thermals is larger than the upward-directed force in the lower part, consistent with the trajectory analysis in section 4 . Overall, $-\partial \overline{p_{\text {dynam }} / \partial z}$ counters roughly from onethird to one-half of the buoyant force, with $-\overline{\partial p_{\text {buoy }} / \partial z}$ countering less than $15 \%$ of the buoyancy. These thermals are therefore sticky, broadly consistent with the theoretical derivation above and with convective thermals in the LESs of Romps and Charn (2015) and Hernandez-Deckers and Sherwood (2016). 
(a) $1000 \mathrm{~m}$ bubble 4-13 min. composite

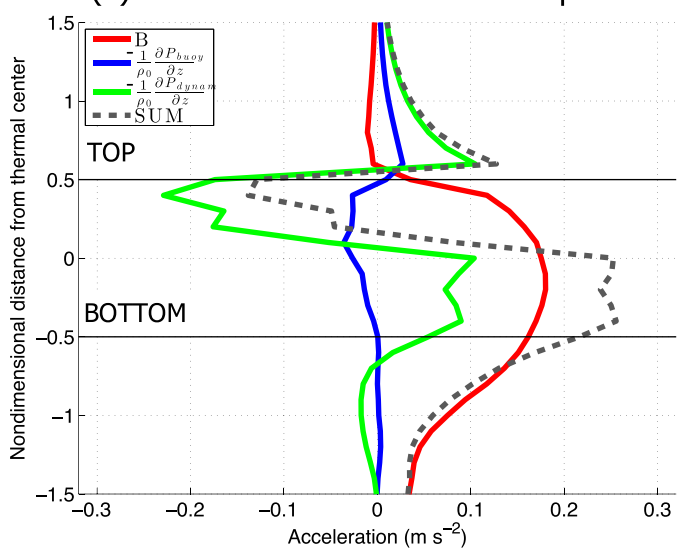

(b) $2000 \mathrm{~m}$ bubble 4-13 min. composite

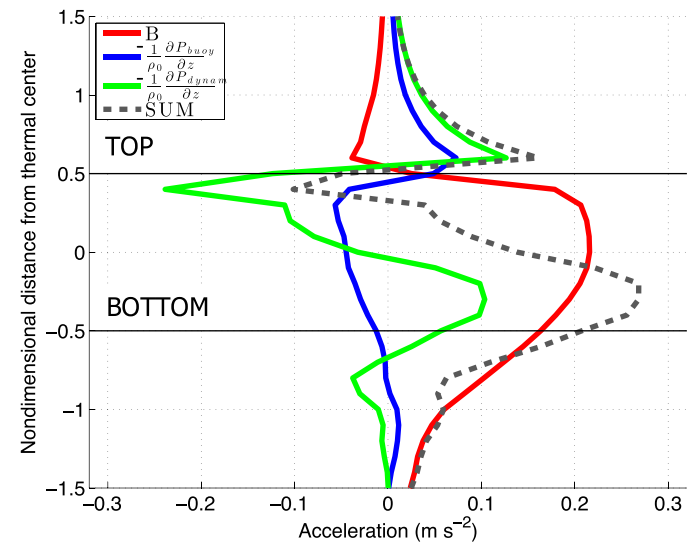

FIG. 12. (a) Composites along the thermal's vertical axis of symmetry $(r=0)$ of $B$ (red line; $\mathrm{ms}^{-2}$ ), $-(1 / \rho)\left(\partial p_{\text {buoy }} / \partial z\right)$ (blue line; $\left.\mathrm{m} \mathrm{s}^{-2}\right),-(1 / \rho)\left(\partial p_{\text {dynam }} / \partial z\right)$ (green line; $\left.\mathrm{m} \mathrm{s}^{-2}\right)$, and the sum of these three terms (gray dashed line) from 4 to $13 \mathrm{~min}$ for the $1000-\mathrm{m}$ bubble simulation. At each time, the depth of the thermal $\Delta H$ is defined as the distance between the top and bottom thermal boundaries, indicated by the horizontal lines labeled TOP and BOTTOM. The normalized height is defined as $Z_{\text {norm }} \equiv\left(z-z_{\text {mid }}\right) / \Delta H$, where $z_{\text {mid }}$ is the height midway through the thermal. Finally, composites are computed using $Z_{\text {norm }}$, where $-0.5<Z_{\text {norm }}<0.5$ defines the boundaries of the thermal. (b) As in (a), but for the 2000-m bubble simulation.

Note that RES is nonnegligible in the $w$ momentum budget. However, this does not necessarily imply that momentum mixing is important; the trajectory analysis of forces discussed in section 4 showed little impact from momentum mixing along the thermal's vertical axis of symmetry, where the model-resolved horizontal velocity is zero. In contrast to the $p_{\text {dynam }}$ and $B$ terms, the sign of RES varies during the simulation and is inconsistent between the 1000- and 2000-m bubble simulations. In addition to contributions from explicit and numerical mixing, the magnitude of RES is likely a result of the model discretization when calculating the terms in (24), calculation of $\partial \widehat{\rho w^{*} / \partial t}$ based on 10-s model output, and shifts in the diagnosed location of the lower thermal boundary.

Overall, theory and simulation results suggest that the buoyancy within thermals induces an asymmetry in the flow that leads to drag primarily from $p_{\text {dynam }}$, in contrast to Hill's vortex, which has a symmetric $p_{\text {dynam }}$ field and zero drag. It is emphasized that this drag is not associated with wave drag, viscous boundary layer effects, or mixing, all of which are neglected in (20)-(23). This drag is manifest as an increased downward-directed $p_{\text {dynam }}$ force on parcels rising along the thermal's central axis in the upper part of the thermal relative to the upwarddirected $p_{\text {dynam }}$ force in the lower part. This counters the increased integrated buoyant forcing experienced by the parcels associated with the thermal's buoyancy. This mechanism of drag is notably different from the drag on a solid sphere moving through a fluid at a high Reynolds number, which is associated with a high-shear boundary layer. A drag via the vertical $p_{\text {dynam }}$ force induced by flow asymmetry from the thermal's buoyancy provides a plausible explanation for why moist thermals typically experience considerable drag and are sticky even when wave drag is minimal as in HernandezDeckers and Sherwood (2016), there is no shear-induced boundary layer, and the buoyant perturbation pressure force is relatively unimportant as in our simulations here. This may also explain why drag in the LES of Hernandez-Deckers and Sherwood (2016, p. 4135) behaved "in a different way to drag on a solid sphere," was not weak, counteracted most of the buoyancy, and was unrelated to mixing.

\section{Implications for convective parameterizations}

As illustrated by Fig. 4, a thermal's core region of upward motion contains its maximum upward velocity $w_{m}^{*}$ roughly consistent with Hill's vortex; $w_{m}^{*}$ generally occurs at a height near the thermal center and is closely associated with the internal toroidal circulation, which does not directly contribute to the net vertical transport of updraft properties. On the other hand, if cloud updraft properties are contained within the thermal boundaries, then the vertical transport of updraft properties is controlled by the thermal ascent rate $w_{t}^{*}$ and size. Since $w_{m}^{*}$ is closely related to the updraft buoyancy profile following (16) as seen in Fig. 8a (see also Peters 2016), and $w_{t}^{*}=\lambda w_{m}^{*}$, the expressions for $\lambda$ and $w_{t}^{*}$ derived in section 2 provide a quantitative way to relate the thermal ascent rate to the updraft buoyancy 
profile. While deep convection schemes typically employ bulk plumes meant to represent the aggregate behavior of an ensemble of convective clouds, for example, in relating entrainment and mass flux profiles, as pointed out by Sherwood et al. (2013, p. 2431), such schemes "must ultimately be founded to some extent on assumptions as to the behavior of individual clouds." Since the vertical mass flux associated with a thermal is equal to its cross-sectional area times its ascent rate, if the ascent rate can be calculated from the updraft buoyancy, then the total vertical convective mass flux (i.e., within a large-scale model grid box) can be directly related to the number and size of convective thermals. This could improve linkages between the vertical velocity and buoyancy within individual convective clouds, the vertical mass flux closure, and assumptions about updraft geometry (e.g., its width).

\section{Summary and conclusions}

In this paper, an analytic expression was derived for the ratio $\lambda$ of the ascent rate of the top of moist deep convective thermals $w_{t}^{*}$ to the maximum vertical velocity within them $w_{m}^{*}$. This expression includes the effects of buoyancy, buoyant perturbation pressure, and dynamic perturbation pressure. The buoyant perturbation pressure was characterized following the analytic formulation of Morrison (2016a) but had little impact on $\lambda$. The dynamic perturbation pressure was assumed to follow the scaling relationship for Hill's analytic spherical vortex. The theoretical $\lambda$ was formulated as a function of two nondimensional buoyancy-related parameters $y$ and $h ; y$ is related to the vertical distribution of buoyancy within the thermal, and $h$ is the ratio of the total buoyancy integrated from the surface to the thermal top and the vertically integrated buoyancy within the thermal.

Theoretical values of $\lambda$ were compared with those directly obtained from numerical simulations. Moist deep convective thermals in the simulations were initiated with warm bubbles of varying size, producing a wide range of updraft sizes and characteristics. The theoretical and simulated values of $\lambda$ showed a reasonable correspondence (correlation coefficient of 0.86) and ranged between $\sim 0.4$ and 0.8 . The largest values of $\lambda$ were associated with $h \sim 1$, meaning that the ratio of the vertically integrated thermal buoyancy to the total vertically integrated buoyancy was relatively large, which occurred early in the simulations. The vertical velocity ratio $\lambda$ tended to decrease over the thermal lifetime. This behavior was captured by the theoretical expressions and is consistent with the thermal laboratory study of Sanchez et al. (1989). Large values of $\lambda$ relative to that for Hill's vortex $(\lambda=0.4)$, which is nonbuoyant, were a direct consequence of positive buoyancy between the height of maximum $w$ within the thermal and the thermal top. This is intuitive since $w$ is fundamentally linked to the buoyant forcing. Thus, the presence of buoyancy within thermals, relative to their environment, fundamentally alters the relationship between the ascent rate of the thermal top and the maximum $w$. This is in contrast to Levine (1959), who suggested that differences in $\lambda$ for real moist convective thermals compared to Hill's vortex value of 0.4 would arise from viscous effects, departures from spherical form, or "small irregularities." On the other hand, vertical gradients of dynamic perturbation pressure explain the decrease of $w$ between the height of maximum $w$, generally occurring near the thermal center, and the thermal top.

The maximum vertical velocity $w_{m}^{*}$ was well estimated by vertically integrating the sum of buoyancy and an estimate of the buoyant perturbation pressure gradient force. In these simulations, the buoyant perturbation pressure was relatively unimportant overall because the positively buoyant updraft regions were generally tall and narrow. Since $w_{t}^{*}$ is equal to $\lambda w_{m}^{*}$, the expressions for $\lambda$ and $w_{m}^{*}$ provide a simple method for quantitatively linking the thermal-top ascent rate to the vertical profile of buoyancy. This is useful since the net vertical transport of updraft properties by thermals is controlled by their ascent rate and size, whereas $w_{m}^{*}$ is more closely related to the thermal's internal toroidal circulation that does not directly contribute to net vertical transport of updraft properties. These expressions can therefore provide a way to more explicitly link the convective vertical mass flux in deep convection parameterizations with updraft properties including ascent rate, vertical velocity and buoyancy profiles, and updraft size.

Because this study focused on the dynamics of moist thermals, we employed a simple treatment of the microphysics that included only cloud liquid condensation and evaporation. However, a more detailed representation of the microphysics could have some impact on the dynamical structure, for example, by altering the buoyancy distribution within thermals via the generation and fallout of precipitation and the formation of cold pools, among others. Conversely, the thermal's dynamical structure will also impact the microphysics. One such interaction pathway is the recycling of liquid and ice hydrometeors through the thermal's toroidal circulation or between successive thermals, which may affect the precipitation efficiency (e.g., Moser and Lasher-Trapp 2017) as well as cloud glaciation (e.g., Lasher-Trapp et al. 2016).

In addition to the impacts on $\lambda$ discussed above, the presence of positive buoyancy within the simulated 
thermals, relative to their environment, also led to a vertical asymmetry in the flow characteristics and dynamic perturbation pressure field, unlike in Hill's vortex, which has a vertically symmetric $p_{\text {dynam }}$ field and zero drag. This asymmetry was associated with a downward drag force from dynamic pressure that counteracted roughly from one-third to one-half of the upward buoyant force, consistent with the "sticky" thermal hypothesis (Romps and Charn 2015; Hernandez-Deckers and Sherwood 2016). Theoretical expressions for this drag were derived that related the drag coefficient $C_{D}$ in the standard drag law to $\lambda$. In contrast to the $p_{\text {dynam }}$ force, the magnitude of the $p_{\text {buoy }}$ force within the thermals was small compared to the buoyancy, with both defined relative to the initial thermodynamic sounding. Overall, these results suggested that the net $p_{\text {dynam }}$ force on a thermal induced by this flow asymmetry can lead to a significant fraction of the buoyant forcing being counteracted, even with negligible wave drag, viscous boundary layer effects, momentum mixing, or buoyant perturbation pressure forcing.

There are some caveats that should be kept in mind when interpreting these results. First, the theoretical expressions for $\lambda$ and $w_{t}^{*}$ neglected the effects of momentum entrainment. However, this assumption is consistent with previous studies showing the limited impact of momentum mixing on the $w$ momentum budget of convective updrafts (e.g., Dawe and Austin 2011; de Roode et al. 2012; Sherwood et al. 2013; Romps and Charn 2015; HernandezDeckers and Sherwood 2016; Morrison 2017). The theoretical expressions also assumed that the buoyancy profile, and hence $y$ and $h$, are known quantities. Buoyancy within updrafts is strongly influenced by entrainment and dilution, which remains a challenge to understand and quantify (de Rooy et al. 2013).

The simple modeling framework utilized here facilitated a direct quantitative comparison with the theoretical expressions. High resolution (100-m horizontal and vertical grid spacings) allowed the model to capture details of the flow including the thermal toroidal circulation. While simple, this framework captured key features of moist convective thermals in previous LESs (Sherwood et al. 2013; Romps and Charn 2015; Hernandez-Deckers and Sherwood 2016; Moser and Lasher-Trapp 2017), including a flow structure similar to Hill's vortex and "stickiness" due to a significant fraction of the upward buoyant forcing that was countered by a downward-directed perturbation pressure gradient force. However, the simulations were not LESs since resolved turbulent motion was limited. LES studies have shown that momentum mixing from turbulent eddies is relatively unimportant in the vertical momentum budget of moist thermals (Sherwood et al. 2013; Romps and Charn 2015; Hernandez-Deckers and
Sherwood 2016). Nonetheless, the effects of explicitly resolving smaller-scale turbulent eddies on $\lambda$ and $w_{t}^{*}$ are unclear, and future work using LES would complement the current study.

Acknowledgments. This material is based on work supported by the National Center of Meteorology, Abu Dhabi, UAE, under the UAE Research Program for Rain Enhancement Science, and also partially supported by the Department of Energy Atmospheric Systems Research (Grant DE-SC0016476). Dr. Richard Rotunno is thanked for providing comments on an earlier version of the manuscript. High-performance computing support on the Yellowstone machine was provided by NCAR's Computer Information Systems Laboratory. The National Center for Atmospheric Research is sponsored by the National Science Foundation.

\section{REFERENCES}

Austin, P. H., M. B. Baker, A. M. Blyth, and J. B. Jensen, 1985: Small-scale variability in warm continental cumulus clouds. J. Atmos. Sci., 42, 1123-1138, https://doi.org/10.1175/15200469(1985)042<1123:SSVIWC > 2.0.CO;2.

Blyth, A. M., and J. Latham, 1993: Development of ice and precipitation in New Mexican summertime cumulus clouds. Quart. J. Roy. Meteor. Soc., 119, 91-120, https://doi.org/ 10.1002/qj.49711950905.

- W. A. Cooper, and J. B. Jensen, 1988: A study of the source of entrained air in Montana cumuli. J. Atmos. Sci., 45, 3944-3964, https://doi.org/10.1175/1520-0469(1988)045<3944: ASOTSO $>2.0 . \mathrm{CO} ; 2$.

Bryan, G. H., and J. M. Fritsch, 2002: A benchmark simulation for moist nonhydrostatic numerical models. Mon. Wea. Rev., 130, 2917-2928, https://doi.org/10.1175/1520-0493(2002)130<2917: ABSFMN $>2.0 . \mathrm{CO} ; 2$.

— J. C. Wyngaard, and J. M. Fritsch, 2003: Resolution requirements for the simulation of deep moist updrafts. Mon. Wea. Rev., 131, 2394-2416, https://doi.org/10.1175/15200493(2003)131<2394:RRFTSO > 2.0.CO;2.

Chow, F. K., R. L. Street, M. Xue, and J. H. Feriger, 2005: Explicit filtering and reconstruction turbulence modeling for large-eddy simulation of neutral boundary layer flow. J. Atmos. Sci., 62, 2058-2077, https://doi.org/10.1175/ JAS3456.1.

Damiani, R., G. Vali, and S. Haimov, 2006: The structure of thermals in cumulus from airborne dual-Doppler radar observations. J. Atmos. Sci., 63, 1432-1450, https://doi.org/10.1175/ JAS3701.1.

Davies-Jones, R., 2003: An expression for effective buoyancy in surroundings with horizontal density gradients. J. Atmos. Sci., 60, 2922-2925, https://doi.org/10.1175/1520-0469(2003)060<2922: AEFEBI $>2.0 . \mathrm{CO} ; 2$.

Dawe, J. T., and P. H. Austin, 2011: The influence of the cloud shell on tracer budget measurements of LES cloud entrainment. J. Atmos. Sci., 68, 2909-2920, https://doi.org/ 10.1175/2011JAS3658.1.

de Roode, S. R., A. P. Siebesma, H. J. J. Jonker, and Y. de Voogd, 2012: Parameterization of the vertical velocity equation for 
shallow convection. Mon. Wea. Rev., 140, 2424-2436, https:// doi.org/10.1175/MWR-D-11-00277.1.

de Rooy, W. C., and Coauthors, 2013: Entrainment and detrainment in cumulus convection: An overview. Quart. J. Roy. Meteor. Soc., 139, 1-19, https://doi.org/10.1002/qj.1959.

Hernandez-Deckers, D., and S. C. Sherwood, 2016: A numerical investigation of cumulus thermals. J. Atmos. Sci., 73, 41174136, https://doi.org/10.1175/JAS-D-15-0385.1.

Heus, T., C. F. J. Pols, H. J. J. Jonker, H. E. A. van den Akker, E. J. Griffith, M. Koutek, and F. H. Post, 2009: A statistical approach to the life cycle analysis of cumulus clouds selected in a virtual reality environment. J. Geophys. Res., 114, D06208, https://doi.org/10.1029/2008JD010917.

Hill, M. J. M., 1894: On a spherical vortex. Philos. Trans. Roy. Soc. London, 185A, 213-245, https://doi.org/10.1098/rsta.1894.0006.

Jeevanjee, N., 2017: Vertical velocity in the gray zone. J. $A d v$. Model. Earth Sci., 9, 2304-2316, https://doi.org/10.1002/ 2017MS001059.

—_, and D. M. Romps, 2016: Effective buoyancy at the surface and aloft. Quart. J. Roy. Meteor. Soc., 142, 811-820, https://doi. org/10.1002/qj.2683.

Jonker, H. J. J., T. Heus, and P. P. Sullivan, 2008: A refined view of vertical mass transport by cumulus convection. J. Geophys. Res., 35, L07810, https://doi.org/10.1029/2007GL032606.

Lamb, H., 1932: Hydrodynamics. Cambridge University Press, 738 pp.

Lasher-Trapp, S., D. C. Leon, P. J. DeMott, C. M. VillanuvaBirriel, A. V. Johnson, D. H. Moser, C. S. Tully, and W. Wu, 2016: A multisensor investigation of rime splintering in tropical maritime cumuli. J. Atmos. Sci., 73, 2547-2564, https:// doi.org/10.1175/JAS-D-15-0285.1.

Lebo, Z. J., and H. Morrison, 2015: Effects of horizontal and vertical grid spacing on mixing in simulated squall lines and implications for convective strength and structure. Mon. Wea. Rev., 143, 4355-4375, https://doi.org/10.1175/MWR-D-150154.1.

Levine, J., 1959: Spherical vortex theory of bubble-like motion in cumulus clouds. J. Meteor., 16, 653-662, https://doi.org/ 10.1175/1520-0469(1959)016<0653:SVTOBL > 2.0.CO;2.

Ludlam, F. H., and R. S. Scorer, 1953: Reviews of modern meteorology-10: Convection in the atmosphere. Quart. J. Roy. Meteor. Soc., 79, 317-341, https://doi.org/10.1002/ qj.49707934102.

Malkus, J. S., and R. S. Scorer, 1955: The erosion of cumulus towers. J. Meteor., 12, 43-57, https://doi.org/10.1175/1520-0469 (1955) $012<0000$ :TEOCT $>2.0$. CO;2.

Morrison, H., 2016a: Impacts of updraft size and dimensionality on the perturbation pressure and vertical velocity in cumulus convection. Part I: Simple, generalized analytic solutions. J. Atmos. Sci., 73, 1441-1454, https://doi.org/10.1175/JAS-D15-0040.1.

2016b: Impacts of updraft size and dimensionality on the perturbation pressure and vertical velocity in cumulus convection. Part II: Comparison of theoretical and numerical solutions. J. Atmos. Sci., 73, 1455-1480, https://doi.org/ 10.1175/JAS-D-15-0041.1.

_ 2017: An analytic description of the structure and evolution of growing deep cumulus updrafts. J. Atmos. Sci., 74, 809-834, https://doi.org/10.1175/JAS-D-16-0234.1.

, J. A. Curry, and V. I. Khvorostyanov, 2005: A new doublemoment microphysics parameterization for application in cloud and climate models. Part I: Description. J. Atmos. Sci., 62, 1665-1677, https://doi.org/10.1175/JAS3446.1.
Morton, B. R., 1957: Buoyant plumes in a moist atmosphere. J. Fluid Mech., 2, 127-144, https://doi.org/10.1017/ S0022112057000038.

— , G. Taylor, and J. S. Turner, 1956: Turbulent gravitational convection from maintained and instantaneous sources. Proc. Roy. Soc. London, 234A, 1-23, https://doi.org/10.1098/ rspa.1956.0011.

Moser, D. H., and S. Lasher-Trapp, 2017: The influence of successive thermals on entrainment and dilution in a simulated cumulus congestus. J. Atmos. Sci., 74, 375-392, https://doi.org/ 10.1175/JAS-D-16-0144.1.

Neggers, R. A. J., A. P. Siebesma, and H. J. J. Jonker, 2002: A multiparcel method for shallow cumulus convection. J. Atmos. Sci., 59, 1655-1668, https://doi.org/10.1175/15200469(2002)059<1655:AMMFSC>2.0.CO;2.

Peters, J. M., 2016: The impact of effective buoyancy and dynamic pressure forcing on vertical velocities within two-dimensional updrafts. J. Atmos. Sci., 73, 4531-4551, https://doi.org/10.1175/ JAS-D-16-0016.1.

Romps, D. M., and A. B. Charn, 2015: Sticky thermals: Evidence for a dominant balance between buoyancy and drag in cloud updrafts. J. Atmos. Sci., 72, 2890-2901, https://doi.org/10.1175/ JAS-D-15-0042.1.

_ , and R. Oktem, 2015: Stereo photogrammetry reveals substantial drag on cloud thermals. Geophys. Res. Lett., 42, 50515057, https://doi.org/10.1002/2015GL064009.

Sanchez, O., D. J. Raymond, L. Libersky, and A. G. Petschek, 1989: The development of thermals from rest. J. Atmos. Sci., 46, 2280-2292, https://doi.org/10.1175/1520-0469(1989)046<2280: TDOTFR $>2.0 . \mathrm{CO} ; 2$.

Scorer, R. S., 1957: Experiments on convection of isolated masses of buoyant fluid. J. Fluid Mech., 2, 583-594, https://doi.org/ 10.1017/S0022112057000397.

_ , and F. H. Ludlam, 1953: Bubble theory of penetrative convection. Quart. J. Roy. Meteor. Soc., 79, 94-103, https://doi.org/ 10.1002/qj.49707933908.

Siebesma, A. P., and J. W. M. Cuijpers, 1995: Evaluation of parametric assumptions for shallow cumulus convection. J. Atmos. Sci., 52, 650-656, https://doi.org/10.1175/15200469(1995)052<0650:EOPAFS $>2.0$.CO;2.

_ , and H. J. J. Jonker, 2000: Anomalous scaling of cumulus cloud boundaries. Phys. Rev. Lett., 85, 214-217, https://doi.org/ 10.1103/PhysRevLett.85.214.

Sherwood, S. C., D. Hernandez-Deckers, and M. Colin, 2013: Slippery thermals and the cumulus entrainment paradox. J. Atmos. Sci., 70, 2426-2442, https://doi.org/10.1175/JAS-D12-0220.1.

Simpson, J., and V. Wiggert, 1969: Models of precipitating cumulus towers. Mon. Wea. Rev., 97, 471-489, https://doi.org/10.1175/ 1520-0493(1969)097<0471:MOPCT>2.3.CO;2.

Smagorinsky, J., 1963: General circulation experiments with the primitive equations. Mon. Wea. Rev., 91, 99-164, https:// doi.org/10.1175/1520-0493(1963)091<0099:GCEWTP>2.3.CO;2.

Stevens, B., and Coauthors, 2001: Simulations of trade wind cumuli under a strong inversion. J. Atmos. Sci., 58, 1870-1891, https:// doi.org/10.1175/1520-0469(2001)058<1870:SOTWCU >2.0.CO;2.

Turner, J. S., 1962: The "starting plume" in neutral surroundings. J. Fluid Mech., 13, 356-368, https://doi.org/10.1017/ S0022112062000762.

_ 1963: Model experiments relating to thermals with increasing buoyancy. Quart. J. Roy. Meteor. Soc., 89, 62-74, https:// doi.org/10.1002/qj.49708937904. 
, 1964: The flow into an expanding thermal. J. Fluid Mech., 18, 195-208, https://doi.org/10.1017/S0022112064000155.

Warren, F. W. G., 1960: Wave resistance to vertical motion in a stratified fluid. J. Fluid Mech., 7, 209-229, https://oi.org/ 10.1017/S0022112060001444.

Weisman, M. L., and J. B. Klemp, 1982: The dependence of numerically simulated convective storms on vertical wind shear and buoyancy. Mon. Wea. Rev., 110, 504-520, https://doi.org/ 10.1175/1520-0493(1982)110<0504:TDONSC > 2.0.CO;2.
Woodward, B., 1959: The motion in and around isolated thermals. Quart. J. Roy. Meteor. Soc., 85, 144-151, https://doi.org/ 10.1002/qj.49708536407.

Yano, J.-I., 2014: Basic convective element: Bubble or plume? A historical review. Atmos. Chem. Phys., 14, 7019-7030, https:// doi.org/10.5194/acp-14-7019-2014.

Zhao, M., and P. H. Austin, 2005: Life cycle of numerically simulated shallow cumulus clouds. Part I: Transport. J. Atmos. Sci., 62, 1269-1290, https://doi.org/10.1175/JAS3414.1. 